\title{
CLASSIFICATION OF K3-SURFACES WITH INVOLUTION AND MAXIMAL SYMPLECTIC SYMMETRY
}

\author{
KRISTINA FRANTZEN
}

\begin{abstract}
АвsтRAст. K3-surfaces with antisymplectic involution and compatible symplectic actions of finite groups are considered. In this situation actions of large finite groups of symplectic transformations are shown to arise via double covers of Del Pezzo surfaces. A complete classification of K3-surfaces with maximal symplectic symmetry is obtained.
\end{abstract}

\section{INTRODUCTION}

An antisymplectic involution $\sigma$ on a complex K3-surface $X$ is a holomorphic involution acting nontrivially on the space $H^{0}\left(X, \Omega^{2}\right)=\mathbb{C} \cdot \omega$ of holomorphic 2-forms on $X$, i.e., $\sigma^{*} \omega=-\omega$. Here we study K3-surfaces with antisymplectic involution from the point of view of symmetry and consider actions of finite groups $G$ of symplectic transformations which are compatible with $\sigma$ in the sense that every $g \in G$ is a holomorphic automorphism of $X$ with $g^{*} \omega=\omega$ and $g \sigma=\sigma g$.

Main results. Given a finite group $G$ we investigate if it can act in a compatible fashion on a K3-surface $X$ with antisymplectic involution $\sigma$. If this is the case, then already the order of $G$ yields strong constraints on the geometry of $X$ and we obtain the following description.

Theorem 2.10. Let $X$ be a K3-surface with a symplectic action of a finite group $G$ centralized by an antisymplectic involution $\sigma$ and assume that $\operatorname{Fix}_{X}(\sigma) \neq \emptyset$. If $|G|>96$, then $X / \sigma$ is a (G-minimal) Del Pezzo surface and Fix $_{X}(\sigma)$ is a smooth connected curve $B$ with $g(B) \geq 3$.

By a theorem due to Mukai [Muk88] finite groups of symplectic transformations on K3-surfaces are characterized by the existence of a certain embedding into a particular Mathieu group and are subgroups of the following eleven finite groups of maximal symplectic symmetry (cf. also [Kon98]).

\begin{tabular}{c|c|c|c|c|c|c|c|c|c|c|c} 
& 1 & 2 & 3 & 4 & 5 & 6 & 7 & 8 & 9 & 10 & 11 \\
\hline group & $L_{2}(7)$ & $A_{6}$ & $S_{5}$ & $M_{20}$ & $F_{384}$ & $A_{4,4}$ & $T_{192}$ & $H_{192}$ & $N_{72}$ & $M_{9}$ & $T_{48}$ \\
\hline order & 168 & 360 & 120 & 960 & 384 & 288 & 192 & 192 & 72 & 72 & 48
\end{tabular}

TABLE 1. Maximal finite groups of symplectic automorphisms on K3-surfaces

This result naturally limits the possible choices of $G$ above and has led us to consider the classification problem for groups $G$ from this list of eleven groups: as a refinement of Theorem 2.10 we obtain the following complete classification.

Theorem 3.1. Let $G$ be a group from Table 1 acting on a K3-surface $X$ by symplectic transformations and let $\sigma$ be an antisymplectic involution on $X$ centralizing $G$ with $\operatorname{Fix}_{X}(\sigma) \neq \emptyset$. Then the pair $(X, G)$ is equivariantly isomorphic to a surface in Table 2 below. In particular, for the groups $G$ numbered 4-8 on Mukai's list, there does not exist a K3-surface with an action of $G \times C_{2}$ satisfying the properties above.

Date: June 10, 2009.

Research supported by Studienstiftung des deutschen Volkes and Deutsche Forschungsgemeinschaft. 


\begin{tabular}{l|l|l} 
& $G$ & K3-surface $X$ \\
\hline $1 a$ & $L_{2}(7)$ & $\left\{x_{1}^{3} x_{2}+x_{2}^{3} x_{3}+x_{3}^{3} x_{1}+x_{4}^{4}=0\right\} \subset \mathbb{P}_{3}$ \\
\hline $1 b$ & $L_{2}(7)$ & Double cover of $\mathbb{P}_{2}$ branched along $\left\{x_{1}^{5} x_{2}+x_{3}^{5} x_{1}+x_{2}^{5} x_{3}-5 x_{1}^{2} x_{2}^{2} x_{3}^{2}=0\right\}$ \\
\hline 2 & $A_{6}$ & $\begin{array}{l}\text { Double cover of } \mathbb{P}_{2} \text { branched along } \\
\left\{10 x_{1}^{3} x_{2}^{3}+9 x_{1}^{5} x_{3}+9 x_{2}^{3} x_{3}^{3}-45 x_{1}^{2} x_{2}^{2} x_{3}^{2}-135 x_{1} x_{2} x_{3}^{4}+27 x_{3}^{6}=0\right\}\end{array}$ \\
\hline $3 a$ & $S_{5}$ & $\left\{\sum_{i=1}^{5} x_{i}=\sum_{i=1}^{6} x_{1}^{2}=\sum_{i=1}^{5} x_{i}^{3}=0\right\} \subset \mathbb{P}_{5}$ \\
\hline $3 b$ & $S_{5}$ & $\begin{array}{l}\text { Minimal desingularization of the double cover of } \mathbb{P}_{2} \text { branched along } \\
\left\{2 x_{1}^{4} x_{2} x_{3}+2 x_{1} x_{2}^{4} x_{3}+2 x_{1} x_{2} x_{3}^{4}-2 x_{1}^{4} x_{2}^{2}-2 x_{1}^{4} x_{3}^{2}-2 x_{1}^{2} x_{2}^{4}-2 x_{1}^{2} x_{3}^{4}\right. \\
-2 x_{2}^{4} x_{3}^{2}-2 x_{2}^{2} x_{3}^{4}+2 x_{1}^{3} x_{2}^{3}+2 x_{1}^{3} x_{3}^{3}+2 x_{2}^{3} x_{3}^{3}+x_{1}^{3} x_{2}^{2} x_{3}+x_{1}^{3} x_{2} x_{3}^{2} \\
\left.+x_{1}^{2} x_{2}^{3} x_{3}+x_{1}^{2} x_{2} x_{3}^{3}+x_{1} x_{2}^{3} x_{3}^{2}+x_{1} x_{2}^{2} x_{3}^{3}-6 x_{1}^{2} x_{2}^{2} x_{3}^{2}=0\right\}\end{array}$ \\
\hline 9 & $N_{72}$ & $\left\{x_{1}^{3}+x_{2}^{3}+x_{3}^{3}+x_{4}^{3}=x_{1} x_{2}+x_{3} x_{4}+x_{5}^{2}=0\right\} \subset \mathbb{P}_{4}$ \\
\hline 10 & $M_{9}$ & Double cover of $\mathbb{P}_{2}$ branched along $\left\{x_{1}^{6}+x_{2}^{6}+x_{3}^{6}-10\left(x_{1}^{3} x_{2}^{3}+x_{2}^{3} x_{3}^{3}+x_{3}^{3} x_{1}^{3}\right)=0\right\}$ \\
\hline $11 a$ & $T_{48}$ & Double cover of $\mathbb{P}_{2}$ branched along $\left\{x_{1} x_{2}\left(x_{1}^{4}-x_{2}^{4}\right)+x_{3}^{6}=0\right\}$ \\
\hline $11 b$ & $T_{48}$ & $\begin{array}{l}\text { Double cover of }\left\{x_{1} x_{2}\left(x_{1}^{4}-x_{2}^{4}\right)+x_{3}^{3}+x_{4}^{2}=0\right\} \subset \mathbb{P}(1,1,2,3) \\
\text { branched along }\left\{x_{3}=0\right\}\end{array}$
\end{tabular}

TABLE 2. K3-surfaces with $G \times C_{2}$-symmetry

In addition to Examples 1a, 3a, 9, 10, and 11a, which have already been described by Mukai (Example 0.4 in [Muk88]) the table contains additional examples (1b, 2, 3b, and 11b) of K3-surfaces with maximal symplectic symmetry. These arise as equivariant double covers of Del Pezzo surfaces.

Remark 1.1. Besides the above mentioned classification of finite groups of symplectic transformations on K3-surfaces by Mukai (cf. also Nikulin's classification in the Abelian case [Nik80] and Kondō's alternative approach using lattice theory [Kon98]) one of our starting points has been the study of K3-surfaces with $L_{2}$ (7)-symmetry in [OZ02]. Although our approach here is independent of the results by Keum, Oguiso, and Zhang (cf. e.g. [OZ02], [KOZ05], [KOZ07], and the summary [Zha07]), our understanding of finite group actions on K3-surfaces strongly gained from the numerous contributions to the subject by the authors above.

We conclude this introduction with an outline of our general classification strategy.

Equivariant minimal model program. The quotient of a K3-surface by an antisymplectic involution $\sigma$ with fixed points centralized by a finite group $G$ is a rational $G$-surface $Y$ to which we apply an equivariant version of the minimal model program respecting finite symmetry groups (cf. Example 2.18 in [KM98] and Section 2.3 in [Mor82]). A detailed exposition using the language of Mori theory and including in particular equivariant analogues of the cone and contraction theorems is given e.g. in [Fra08], Chapter 2 and [FH09], Section 4. Here we briefly summarize the program in the following classification result.

Proposition 1.2. Let $Y$ be a smooth projective surface with an action of a finite group $G$. Then there exists a sequence of G-equivariant extremal contractions $Y \rightarrow Y_{(1)} \rightarrow \cdots \rightarrow Y_{\min }$ such that $Y_{\min }$ satisfies one of the following conditions:

(1) The canonical line bundle $\mathscr{K}_{Y_{\min }}$ is nef.

(2) $Y_{\min }$ is an G-equivariant conic bundle over a smooth curve, i.e., there exists an G-equivariant morphism $f: Y_{\min } \rightarrow C$ onto a smooth curve $C$ such that the general fiber is a rational curve. If $f$ has singular fibers, these consist of two (-1)-curves intersecting transversally. 
(3) $\mathscr{K}_{Y_{\min }}^{-1}$ is ample, i.e., $Y_{\min }$ is a Del Pezzo surface.

Each extremal contraction is the contraction of finitely many disjoint (-1)-curves forming a G-orbit.

The surface $Y_{\min }$ is referred to as a G-minimal model of $Y$, and the map $Y \rightarrow Y_{\min }$ is called a Mori reduction. If $Y$ is a rational surface, then $Y_{\min }$ is either a Del Pezzo surface or an equivariant conic bundle over $\mathbb{P}_{1}$, i.e., the reduction leads to the well-known classification of $G$-minimal rational surfaces ([Man67], [Isk80]).

Remark 1.3. Equivariant Mori reduction and the theory of $G$-minimal models have applications in various different contexts and can also be generalized to higher dimensions. Initiated by Bayle and Beauville in [ $\mathrm{BaBe00}]$, the methods have been employed in the classification of subgroups of the Cremona group $\operatorname{Bir}\left(\mathbb{P}_{2}\right)$ for example by Beauville and Blanc ([Bea07], [BeBl04], [Bla06] [Bla07]), de Fernex [dF04], Dolgachev and Iskovskikh [DI06], [DI07], and Zhang [Zha01]. These references also provide details regarding the equivariant minimal model program summarized above.

Del Pezzo surfaces. In all cases under consideration in this article the surface $Y_{\min }$ is a Del Pezzo surface. Del Pezzo surfaces are classified according to their degree $1 \leq d \leq 9$, which is by definition the selfintersection number of the canonical divisor class. A Del Pezzo surface is either obtained from $\mathbb{P}_{2}$ by blowing up $9-d$ points in general position or is isomorphic to $\mathbb{P}_{1} \times \mathbb{P}_{1}$. The anticanonical map realizes a Del Pezzo surface of degree $d \geq 3$ as a degree $d$ subvariety in $\mathbb{P}_{d}$, defines a double cover for $d=2$, and an elliptic fibration for $d=1$.

Our understanding of Del Pezzo surfaces as surfaces obtained by blowing up points in $\mathbb{P}_{2}$ or as degree $d$ subvarieties of $\mathbb{P}_{d}$ enables us to decide whether a given finite group $G$ can occur as a subgroup of the automorphisms group $\operatorname{Aut}(Z)$ of a certain Del Pezzo surface $Z$. Furthermore, we repeatedly consider the configuration of (-1)-curves on a Del Pezzo surfaces $Z$ and the induced action of $\operatorname{Aut}(Z)$ on it. In certain cases our analysis relies on Dolgachev's discussion of automorphism groups of Del Pezzo surfaces in [Dol09], Chapter 10.

Using detailed knowledge of the equivariant reduction map $Y \rightarrow Y_{\min }$, the structure of the invariant set $\operatorname{Fix}_{X}(\sigma)$, and the equivariant geometry of Del Pezzo surfaces, we classify $Y_{\min }, Y$, and $\operatorname{Fix}_{X}(\sigma)$ and can describe $X$ as an equivariant double cover of a possibly blown-up Del Pezzo surface.

Acknowledgements. The results presented in this note originate from the author's Ph.D. thesis at RuhrUniversität Bochum. The author would like to thank Alan Huckleberry for his advice and support and for numerous intensive, helpful discussions.

\section{Centralizers of antisymplectic inVolutions}

This section is dedicated to a rough classification (Theorem 2.10) of K3-surfaces with antisymplectic involutions centralized by large groups of symplectic transformations. For a group $H<\operatorname{Aut}(X)$ we denote by $H_{\text {symp }} \triangleleft H$ the normal subgroup of symplectic automorphisms. The group $H / H_{\text {symp }}$ acts effectively on $\Omega^{2}(X)=\mathbb{C} \cdot \omega_{X}$. In particular, if $H$ is finite, then $H / H_{\text {symp }}$ is cyclic.

We consider a K3-surface $X$ with an action of a finite group $G \times C_{2}<\operatorname{Aut}(X)$ and assume that $G<$ $\mathrm{Aut}_{\text {symp }}(X)$ whereas $C_{2}$ is generated by an antisymplectic involution $\sigma$ centralizing $G$. Furthermore, we assume that $\operatorname{Fix}_{X}(\sigma) \neq \emptyset$. Let $\pi: X \rightarrow X / \sigma=Y$ denote the quotient map, $R=\operatorname{Fix}_{X}(\sigma)$ and $B=\pi(R)$ the ramification and branch set of $\pi$. The quotient surface $Y$ is a smooth rational $G$-surface to which we apply an equivariant minimal model program. By Proposition 1.2 a $G$-minimal model $Y_{\min }$ of $Y$ is either a Del Pezzo surface or an equivariant conic bundle over $\mathbb{P}_{1}$. In the later case, the following lemma shows that the possibilities for $G$ are limited by the classification of finite groups with an effective action on $\mathbb{P}_{1}$, i.e., cyclic and dihedral groups, $C_{n}$ and $D_{2 n}$, and the exceptional tetrahedral, octahedral, and icosahedral group, $T_{12}, O_{24}, I_{60}$. 
Remark 2.1. Several times we will use the fact that the order of a symplectic transformation on a K3surfaces is at most eight. This follows implicitly from Table 1, but is originally due to an argument of Nikulin classifying finite Abelian groups of symplectic transformation on K3-surfaces in [Nik80, §5]. Note that Nikulin' approach also shows that a symplectic automorphism of order $2,3,4,5,6,7,8$ has $8,6,4,4,2,3,2$ fixed points, respectively.

Lemma 2.2. If a $G$-minimal model $Y_{\min }$ of $Y$ is an equivariant conic bundle, then $|G| \leq 96$.

Proof. Let $\varphi: Y_{\min } \rightarrow \mathbb{P}_{1}$ be an equivariant conic bundle structure on $Y_{\min }$. We consider the induced action of $G$ on the base $\mathbb{P}_{1}$. If this action is effective, then $G$ is among the groups specified above. Since the maximal order of an element in $G$ is eight, it follows that the order of $G$ is bounded by 60 .

If the action of $G$ on the base $\mathbb{P}_{1}$ is not effective, every element $n$ of the ineffectivity $N<G$ has two fixed points in the general fiber, which is isomorphic to $\mathbb{P}_{1}$. This gives rise to a positive-dimensional $n$-fixed point set in $Y_{\min }$ and $Y$. A symplectic automorphism however has only isolated fixed points. It follows that the action of $n$ coincides with the action of $\sigma$ on $\pi^{-1}\left(\operatorname{Fix}_{Y}(N)\right) \subset X$ and the order of $n$ is two. Since $N$ acts effectively on the general fiber, it follows that $N$ is isomorphic to either $C_{2}$ or $C_{2} \times C_{2}$.

If $G / N$ is isomorphic to the icosahedral group $I_{60}=A_{5}$, then $G$ fits into the exact sequence $1 \rightarrow N \rightarrow$ $G \rightarrow A_{5} \rightarrow 1$ for $N=C_{2}$ or $C_{2} \times C_{2}$. Let $\xi$ be an element of order five in $G$. Since neither $C_{2}$ nor $C_{2} \times C_{2}$ has automorphisms of order five it follows that $\xi$ centralizes the normal subgroup $N$. In particular, there is a subgroup $C_{2} \times C_{5} \cong C_{10}$ in $G$ which contradicts the assumption that $G$ is a group of symplectic transformations and therefore its elements have order at most eight.

If $G / N$ is cyclic or dihedral, we again use the fact that the order of elements in $G$ is bounded by 8 to conclude $|G / N| \leq 16$. It follows that the maximal possible order of $G / N$ is $\left|O_{24}\right|=24$. Using $|N| \leq 4$ we obtain $|G| \leq 96$.

For $|G|>96$ the lemma above allows us to restrict our classification to the case where any $G$-minimal model $Y_{\min }$ of $Y$ is a Del Pezzo surface.

2.1. Branch curves and Mori fibers. We use the term curve for irreducible 1-dimensional analytic subsets of surfaces. For the remainder of this section we fix an equivariant Mori reduction $M: Y \rightarrow Y_{\min }$. A rational curve $E \subset Y$ is called a Morifiber if it is contracted in some step of the equivariant Mori reduction $Y \rightarrow Y_{\min }$. The set of all Mori fibers is denoted by $\mathscr{E}$. Its cardinality $|\mathscr{E}|$ is denoted by $m$.

A linearization argument shows that $\operatorname{Fix}_{X}(\sigma)$ is a disjoint union of smooth curves. More precisely, by a basic result originally due to Nikulin ([Nik83]), the set $\operatorname{Fix}_{X}(\sigma)$ is either a union of two linearly equivalent elliptic curves or the union of one single curve of arbitrary genus with a (possibly empty) union of rational curves. In the following we let $n$ denote the total number of rational curves in $\operatorname{Fix}_{X}(\sigma)$. We choose a triangulation of $\operatorname{Fix}_{X}(\sigma)$ and extend it to a triangulation of the surface $X$. The topological Euler characteristic of the double cover is given by

$$
e(X)=24=2 e(Y)-\sum_{C \subset \mathrm{Fix}_{X}(\sigma)} e(C) \geq 2 e(Y)-2 n=2\left(e\left(Y_{\min }\right)+m\right)-2 n .
$$

This yields $m \leq n+12-e\left(Y_{\min }\right)$. Combining this with $e\left(Y_{\min }\right) \geq 3$ shows that the total number $m$ of Mori fibers in $Y$ is bounded by

$$
m \leq n+12-e\left(Y_{\min }\right) \leq n+9 .
$$

Remark 2.3. In the following, we repeatedly use the fact that for a finite proper surjective holomorphic map of complex manifolds (spaces) $\pi: X \rightarrow Y$ of degree $d$, the intersection number of pullback divisors fulfills $\left(\pi^{*} D_{1} \cdot \pi^{*} D_{2}\right)=d\left(D_{1} \cdot D_{2}\right)$. In particular, if $\pi: X \rightarrow Y$ is a degree two map of surfaces, the image $\pi(C) \subset Y$ of a curve $C \subset X$ contained in the ramification locus $R$ of $\pi$ has self-intersection $(\pi(C))^{2}=2 C^{2}$. 
Lemma 2.4. Every Mori fiber $E \in \mathscr{E}, E \not \subset B$ meets the branch locus $B$ in at most two points. If $E$ and $B$ are tangent at $p$, then $E \cap B=\{p\}$ and $(E \cdot B)_{p}=2$.

Proof. Let $E \in \mathscr{E}, E^{2}=k$ be a Mori fiber such that $E \not \subset B$ and $|E \cap B| \geq 2$ or $E \cdot B \geq 3$. By the remark above, the divisor $\pi^{-1}(E)=\pi^{*} E$ has self-intersection $2 k$. Assume that $\pi^{-1}(E)$ is reducible and let $\tilde{E}_{1}, \tilde{E}_{2}$ denote its irreducible components. These are rational and therefore, by adjunction on the K3-surface $X$, have self-intersection number -2 . We write

$$
0>2 k=\left(\pi^{-1}(E)\right)^{2}=\tilde{E}_{1}^{2}+\tilde{E}_{2}^{2}+2\left(\tilde{E}_{1} \cdot \tilde{E}_{2}\right)=-4+2\left(\tilde{E}_{1} \cdot \tilde{E}_{2}\right) .
$$

Since $\tilde{E}_{1}$ and $\tilde{E}_{2}$ intersect at points in the preimage of $E \cap B$, we obtain $\tilde{E}_{1} \cdot \tilde{E}_{2} \geq 2$, a contradiction. It follows that $\pi^{-1}(E)$ is irreducible. Consequently, $k=-1$ and $\pi^{-1}(E)$ is a smooth rational curve with precisely two $\sigma$-fixed points showing $|E \cap B|=2$. It remains to show that the intersection is transversal.

To see this, let $N_{\tilde{E}}$ denote the normal bundle of $\tilde{E}$ in $X$. We consider the induced action of $\sigma$ on $N_{\tilde{E}}$ by a bundle automorphisms. We may equivariantly identify a tubular neighbourhood of $\tilde{E}$ in $X$ with $N_{\tilde{E}}$ via a $C^{\infty}$-diffeomorphism. The $\sigma$-fixed point curves intersecting $\tilde{E}$ map to curves of $\sigma$-fixed points in $N_{\tilde{E}}$ intersecting the zero-section and vice versa. Let $D$ be a curve of $\sigma$-fixed points in $N_{\tilde{E}}$. If $D$ is not a fiber of $N_{\tilde{E}}$, it follows that $\sigma$ stabilizes all fibers intersecting $D$ and the induced action of $\sigma$ on the base must be trivial, a contradiction. It follows that the $\sigma$-fixed point curves correspond to fibers of $N_{\tilde{E}}$, and $E$ and $B$ meet transversally.

By negation of the implication above, if $E$ and $B$ are tangent at $p$, then $|E \cap B|=1$ and $E \cdot B=2$.

Remark 2.5. Adjunction on $X$ and elementary considerations involving intersection numbers resembling those in the proof of the lemma above yield the following summary of the possible intersection geometry of a Mori fiber $E \in \mathscr{E}$ with the branch locus $B$ in relation to the self-intersection number of $E$.

If $E \subset B$, then $E^{2}=-4$. If $E \cap B=\emptyset$, then $E^{2}=-2$ and $\pi^{-1}(E)$ is reducible. If $E \cap B=\{p\}$, then $E^{2}=-1$ and $\pi^{-1}(E)$ is reducible. If $E \cap B=\left\{p_{1}, p_{2}\right\}$, then $E^{2}=-1$ and $\pi^{-1}(E)$ is irreducible. More generally, the arguments involved apply to any (-1)-curve $E$ on $Y$ and therefore $E$ meets $B$ in either one or two points and $\pi^{-1}(E)$ is reducible or irreducible, respectively.

2.2. Rational branch curves. In this subsection we find conditions on $G$, in particular conditions on the order of $G$, guaranteeing the absence of rational curves in $\operatorname{Fix}_{X}(\sigma)$.

It follows from adjunction that a curve with negative self-intersection on a Del Pezzo surface necessarily has self-intersection -1. So if $Y_{\min }$ is a Del Pezzo surface, all rational branch curves of $\pi$, which have self-intersection -4 by Remark 2.3, need to be modified by the Mori reduction when passing to $Y_{\min }$ and therefore have nonempty intersection with the union of Mori fibers. We benefit from the following elementary observation regarding the behaviour of self-intersection numbers under monoidal transformations.

Remark 2.6. Let $\tilde{X}$ and $X$ be smooth projective surfaces and let $b: \tilde{X} \rightarrow X$ be the blow-down of a (-1)-curve $E \subset \tilde{X}$. For a curve $B \subset \tilde{X}, B \neq E$, the self-intersection of its image in $X$ is given by $(b(B))^{2}=B^{2}+(E \cdot B)^{2}$.

We denote by $\mathscr{C}$ the set of rational branch curves of $\pi$. The total number $|\mathscr{C}|$ of these curves is denoted by $n$. The union of all Mori fibers not contained in the branch locus $B$ is denoted by $\bigcup E_{i}$. Let $\mathscr{C}_{\geq k}=\{C \in$ $\left.\mathscr{C}|| C \cap \bigcup E_{i} \mid \geq k\right\}$ be the set of those rational branch curves $C$ which meet $\bigcup E_{i}$ in at least $k$ distinct points and set $r_{k}=\left|\mathscr{C}_{\geq k}\right|$. We denote by $\mathscr{E}_{\geq k}$ the set of Mori fibers $E \not \subset B$ which intersect some $C \in \mathscr{C}_{\geq k}$ and define

$$
P_{k}=\left\{(p, E) \mid p \in C \cap E, E \in \mathscr{E}_{\geq k}, C \in \mathscr{C}_{\geq k}\right\} \subseteq Y \times \mathscr{E}_{\geq k}
$$

and the projection map $\operatorname{pr}_{k}: P_{k} \rightarrow \mathscr{E}_{\geq k}$ mapping $(p, E)$ to $E$. This map is surjective by definition of $\mathscr{E}_{\geq k}$ and its fibers consist of $\leq 2$ points by Lemma 2.4. Using $\left|P_{k}\right| \geq k r_{k}$ we see

$$
\left|\mathscr{E}_{\geq k}\right| \geq \frac{k}{2} r_{k}
$$


Let $N$ be the largest positive integer such that $\mathscr{C}_{\geq N}=\mathscr{C}$, i.e., each rational ramification curve is intersected at least $N$ times by Mori fibers. A curve $C \in \mathscr{C}$ which is intersected precisely $N$ times by Mori fibers is referred to as a minimizing curve. In the following, let $C$ be a minimizing curve and let $H=\operatorname{Stab}_{G}(C)<G$ be the stabilizer of $C$ in $G$. The index of $H$ in $G$ is bounded by $n=r_{N}$.

The rational curves in $\operatorname{Fix}_{X}(\sigma)$ generate a sublattice of $\operatorname{Pic}(X)$ of signature $(0, n)$ and it therefore follows immediately that $n \leq 19$. A sharper bound $n \leq 16$ for the number of disjoint (-2)-curves on a K3-surface has been obtained by Nikulin [Nik76]. In our setup an even sharper bound is due to Zhang [Zha98, Theorem 3], stating that the total number of connected curves in the fixed point set of an antisymplectic involution on a K3-surface is bounded by 10. In the following, we use Zhang's bound for the number $n$ of rational curves in $\operatorname{Fix}_{X}(\sigma)$,

$$
n \leq 10 .
$$

Note, however, that all results can likewise be obtained by using the weakest bound $n \leq 19$. For $N \geq 4$ Zhang's bound can be sharpened using the notion of Mori fibers and minimizing curves. Using inequalities (1) and (2) we find

$$
\frac{N}{2} n=\frac{N}{2} r_{N} \leq\left|\mathscr{E}_{\geq N}\right| \leq|\mathscr{E}| \leq n+12-e\left(Y_{\min }\right) \leq n+9
$$

In the following we consider the stabilizer $H$ of a minimizing curve $C$ and using the above inequalities for $n$, we obtain bounds for the order $|G|$ of $G$ guaranteeing the absence of rational curves in $\operatorname{Fix}_{X}(\sigma)$.

Proposition 2.7. Let $X$ be a K3-surface with an action of a finite group $G \times\langle\sigma\rangle$ such that $G<\operatorname{Aut}_{\text {symp }}(X)$ and $\sigma$ is an antisymplectic involution with fixed points. If $|G|>108$, then $\operatorname{Fix}_{X}(\sigma)$ does not contain rational curves.

Proof. Let $|G|>108$ and assume on the contrary that $\operatorname{Fix}_{X}(\sigma)$ contains rational curves. We consider a minimizing curve $C \subset B$ and its stabilizer $\operatorname{Stab}_{G}(C)=: H$. Since a symplectic automorphism on $X$ does not admit a one-dimensional set of fixed points, it follows that the action of $H$ on $C$ is effective and $H$ is one the classical finite groups $C_{n}, D_{2 n}, T_{12}, O_{24}, I_{60}$. We recall the possible lengths of $H$-orbits in $C$ : the length of an orbit of a dihedral group is at least two, the length of a $T_{12}$-orbit in $\mathbb{P}_{1}$ is at least four, the length of an $\mathrm{O}_{24}$-orbit in $\mathbb{P}_{1}$ is at least six, and the length of an $I_{60}$-orbit in $\mathbb{P}_{1}$ is at least twelve.

Let $Y_{\min }$ be a $G$-minimal model of $X / \sigma=Y$. Recall that by Lemma 2.2 the surface $Y_{\min }$ is a Del Pezzo surface. Each rational branch curve is a (-4)-curve in $Y$. Since its image in $Y_{\min }$ has self-intersection $\geq-1$, it must intersect Mori fibers.

- If $N=1$, i.e., the rational curve $C$ meets the union of Mori fibers in exactly one point $p$, then $p$ is a fixed point of the $H$-action on $C$. In particular, $H$ is a cyclic group $C_{k}$ and $k \leq 8$ by Remark 2.1 . Since the index of $H$ in $G$ is bounded by $n \leq 10$, it follows that $|G| \leq 80$.

- If $N=2$, then $H$ is either a cyclic or a dihedral group. By Proposition 3.10 in [Muk88] the maximal order of a dihedral group of symplectic automorphisms on a K3-surface is 12 . We first assume $H \cong D_{2 m}$ and that the $G$-orbit $G$.C of the rational branch curve $C$ has the maximal length $n=|G . C|=10$, i.e., $B=$ G.C. Each curve in G.C meets the union of Mori fibers in precisely two points forming an $D_{2 m}$-orbit. If a Mori fiber $E_{C}$ meets the curve $C$ twice, then it follows from Lemma 2.4 that $E$ meets no other curve in $B$. The contraction of $E$ transforms $C$ into a singular curve of self-intersection zero. The Del Pezzo surface $Y_{\min }$ does however not admit a curve of this type. It follows, that $E$ meets a Mori fiber $E^{\prime}$ which is contracted in a later step of the Mori reduction and meets no other Mori fiber than $E^{\prime}$. The described configuration G.E $\cup G . E^{\prime}$ requires a total number of at least 20 Mori fibers and therefore contradicts inequality (1). If $C$ meets two distinct Mori fibers $E_{1}, E_{2}$, each of these two can meet at most one further curve in $B$. The contraction of $E_{1}$ and $E_{2}$ transforms $C$ into a (-2)-curve. As above, the existence of further Mori 
fibers meeting $E_{i}$ follows. Again, by invariance, the total number of Mori fibers exceeds 20, a contradiction. It follows that either $H$ is cyclic or $|G . C| \leq 9$. Both cases imply $|G| \leq 108$.

- If $N=3$, let $S=\left\{p_{1}, p_{2}, p_{3}\right\}$ be the points of intersection of $C$ with the union $\bigcup E_{i}$ of Mori fibers. The set $S$ is $H$-invariant. It follows that $H$ is either trivial or isomorphic to $C_{2}, C_{3}$ or $D_{6}$ and that $|G| \leq 60$

- If $N=4$, it follows from inequality (4) that $n \leq 9$. Now $|H| \leq 12$ implies $|G| \leq 108$.

- If $N=5$, the largest possible group acting on $C$ such that there is an invariant subset of cardinality 5 is the dihedral group $D_{10}$. Inequality (4) implies $n \leq 6$, we conclude $|G| \leq 60$.

- If $N=6$, then $n \leq 4$ and $|H| \leq 24$ implies $|G| \leq 96$.

- If $N \geq 12$, then $n=1$ and $H=G$. The maximal order 60 is attained by the icosahedral group.

- If $6<N<12$, we combine $n \leq 4$ and $|H| \leq 24$ to obtain $|G| \leq 96$.

The case by case discussion shows that the existence of a rational curve in $B$ implies $|G| \leq 108$ and contradicts our assumption. The proposition follows.

Remark 2.8. If the group $G$ under consideration is known not to contain certain subgroups (such as large dihedral groups or $T_{12}, O_{24}$ or $I_{60}$ ), then the condition $|G|>108$ in the proposition above can be improved and non-existence of rational ramification curves follows even for smaller groups $G$ (cf. Lemmata 3.26 , 3.28 , and 3.32).

2.3. Elliptic branch curves. The aim of this section is to determine conditions on the order of $G$ which allow us to exclude elliptic curves in $\operatorname{Fix}_{X}(\sigma)$. We prove:

Proposition 2.9. Let $X$ be a K3-surface with an action of a finite group $G \times\langle\sigma\rangle$ such that $G<\operatorname{Aut}_{\text {symp }}(X)$ and $\sigma$ is an antisymplectic involution with fixed points. If $|G|>108$, then $\operatorname{Fix}_{X}(\sigma)$ contains neither rational nor elliptic ramification curves.

Proof. By the preceding proposition $\operatorname{Fix}_{X}(\sigma)$ does not contain rational curves. It follows from Nikulin's description of $\operatorname{Fix}_{X}(\sigma)$ in [Nik83] that it is either a single curve of genus $g \geq 1$ or the disjoint union of two elliptic curves.

Let $T \subset B$ be an elliptic branch curve and let $H:=\operatorname{Stab}_{G}(T)$. If $H \neq G$, then $H$ has index two in $G$. The action of $H$ on $T$ is effective. After conjugation the subgroup $H<\operatorname{Aut}(T)$ inherits the semidirect product structure of $\operatorname{Aut}(T)=L \ltimes T$ for $L \in\left\{C_{2}, C_{4}, C_{6}\right\}$. I.e.,

$$
H=(H \cap L) \ltimes(H \cap T) .
$$

We refer to this decomposition as the normal form of $H$. By Lemma 2.2 any $G$-minimal model of $Y$ is a Del Pezzo surface and therefore, by adjunction, does not admit elliptic curves with self-intersection zero. It follows that $T$ meets the union $\bigcup E_{i}$ of Mori fibers. Let $E$ be a Mori fiber meeting $T$. By Lemma 2.4their intersections fulfills $|T \cap E| \in\{1,2\}$. Since the total number of Mori fibers is bounded by 9 (cf. inequality (1D), the index of the stabilizer $\operatorname{Stab}_{H}(E)$ of $E$ in $H$ is bounded by 9. If $T \cap E=\{p\}$, then $\operatorname{Stab}_{H}(E)$ is a cyclic group of order less than or equal to six and it follows that $|G| \leq 6 \cdot 9 \cdot 2=108$. If $T \cap E=\left\{p_{1}, p_{2}\right\}$, then $B \cap E=T \cap E$ and the stabilizer $\operatorname{Stab}_{G}(E)$ of $E$ in $G$ is contained in $H$. If both points $p_{1}, p_{2}$ are fixed by $\operatorname{Stab}_{G}(E)$, then $\left|\operatorname{Stab}_{G}(E)\right| \leq 6$. If $p_{1}, p_{2}$ form a $\operatorname{Stab}_{G}(E)$-orbit, then in the normal form $\left|\operatorname{Stab}_{G}(E) \cap T\right|=2$. It follows that $\operatorname{Stab}_{G}(E)$ is either $C_{2}$ or $D_{4}$. The index of $\operatorname{Stab}_{G}(E)$ in $G$ is bounded by 9 and $|G| \leq 54$.

In summary, the existence of an elliptic curve in $B$ implies $|G| \leq 108$.

2.4. Rough classification. With the preparations of the previous subsections we may now turn to a classification result for K3-surfaces with antisymplectic involution centralized by a large group.

Theorem 2.10. Let $X$ be a K3-surface with a symplectic action of a finite group $G$ centralized by an antisymplectic involution $\sigma$ such that $\operatorname{Fix}_{X}(\sigma) \neq \emptyset$. If $|G|>96$, then $X / \sigma$ is a $G$-minimal Del Pezzo surface and Fix $_{X}(\sigma)$ is a smooth connected curve $B$ with $g(B) \geq 3$. 
KRISTINA FRANTZEN

Proof. The group $G$ is a subgroup of one of the eleven maximal finite groups of symplectic transformations on Mukai's list [Muk88] (cf. Table 1). None of these groups can have a subgroup $G$ with $96<|G|<120$. In particular, the order of $G$ is at least 120 . We may therefore apply the results of the previous two sections and conclude that $\pi: X \rightarrow X / \sigma=Y$ is branched along a single smooth curve $B$ of general type. Its genus $g(B)$ must be greater than or equal to three by Hurwitz's formula. It remains to show that $Y$ is $G$-minimal. Assume on the contrary that $Y$ is not $G$-minimal. Then there exists a Mori fiber $E \subset Y$ with $E^{2}=-1$. By Remark 2.5 it intersects the branch curve $B$ in one or two points. Let $\operatorname{Stab}_{G}(E)$ denote the stabilizer of $E$ in G.

If $\pi^{-1}(E)$ is reducible its two irreducible components meet transversally in one point corresponding to $\{p\}=E \cap B$. The curve $E$ is tangent to $B$ at $p$ and we consider the linearization of the action of $\operatorname{Stab}_{G}(E)$ at $p$. If the action of $\operatorname{Stab}_{G}(E)$ on $E$ is not effective, the linearization of the ineffectivity $I<\operatorname{Stab}_{G}(E)$ yields a trivial action of $I$ on the tangent line of $B$ at $p$. It follows that the action of $I$ is trivial in a neighbourhood of $\pi^{-1}(p) \in R=\pi^{-1}(B)$. This is contrary to the assumption that $G$ acts symplectically on $X$. Consequently, the action of $\operatorname{Stab}_{G}(E)$ on $E$ is effective and in particular, $\operatorname{Stab}_{G}(E)$ is a cyclic group.

If $\pi^{-1}(E)$ is irreducible, then it is a smooth rational curve with an effective action of $\operatorname{Stab}_{G}(E)$. It follows that $\operatorname{Stab}_{G}(E)$ is either cyclic or dihedral.

We conclude that the order of $\operatorname{Stab}_{G}(E)$ is bounded by 12 and the index of $G_{E}$ in $G$ is strictly greater than nine. By inequality (1) the total number $m$ of Mori fibers however satisfies $m \leq 9$. This contradiction shows that $Y$ is $G$-minimal and, in particular, a Del Pezzo surface.

Remark 2.11. Let $X$ be a K3-surface with a symplectic action of $G$ centralized by an antisymplectic involution $\sigma$ with $\operatorname{Fix}_{X}(\sigma) \neq \emptyset$ and let $E$ be a (-1)-curve on $Y=X / \sigma$. Then the argument above can be applied to see that the stabilizer of $E$ in $G$ is cyclic or dihedral and therefore has order at most 12 .

In the following section, the classification above is applied and extended to the case where $G$ is a maximal group of symplectic transformations on a K3-surface.

\section{MAXimal Finite GROUPS OF SYMPLECTIC TRANSFORMATIONS}

In this section we consider K3-surfaces with a symplectic action of one of the eleven groups from Mukai's list (Table 1) centralized by an antisymplectic involution and prove the following classification result.

Theorem 3.1. Let $G$ be a group from Table 1 acting on a K3-surface $X$ by symplectic transformations and $\sigma$ be an antisymplectic involution on $X$ centralizing $G$ with $\operatorname{Fix}_{X}(\sigma) \neq \emptyset$. Then the pair $(X, G)$ is equivariantly isomorphic to a surface in Table 2 In particular, for the groups $G$ numbered 4-8 on Mukai's list, there does not exist a K3-surface with an action of $G \times C_{2}$ satisfying the properties above.

For the proof of this theorem we consider each group separately, show that any $G$-minimal model $Y_{\min }$ of the quotient surface $Y=X / \sigma$ is a Del Pezzo surface, and investigate which Del Pezzo surfaces admit an action of the group $G$.

It is then essential to study the branch locus $B$ of the covering $X \rightarrow Y$. As a first step, we exclude rational and elliptic curves in $B$ by studying their images in $Y_{\min }$ and their intersection with the union of Mori fibers. We then deduce that $B$ consists of a single curve of genus $\geq 2$ with an effective action of the group $G$. The possible genera of $B$ are restricted by the nature of the group $G$ and the Riemann-Hurwitz formula for the quotient of $B$ by an appropriate normal subgroup $N$ of $G$. The equations of $B$ or $X$ given in Table 2 are derived using invariant theory.

Throughout the remainder of this chapter, the Euler characteristic formula

$$
24=e(X)=2 e\left(Y_{\min }\right)+2 m-2 n+\underbrace{(2 g-2)}_{\text {if branch curve with } g \geq 2 \text { exists }}
$$


is exploited at various points. Here $m$ denotes the total number of Mori contractions of the reduction $Y \rightarrow Y_{\min }$, the total number of rational branch curves is denoted by $n$ and $g$ is the genus of a non-rational branch curve.

Equivariant equivalence. Let us briefly formalize the notion of equivariant equivalence.

Definition 3.2. Let $\left(X_{1}, \sigma_{1}\right)$ and $\left(X_{2}, \sigma_{2}\right)$ be K3-surfaces with antisymplectic involution and let $G$ be a finite group acting on $X_{1}$ and $X_{2}$ by $\alpha_{i}: G \rightarrow \operatorname{Aut}_{\text {symp }}\left(X_{i}\right)$, such that $\alpha_{i}(g) \circ \sigma_{i}=\sigma_{i} \circ \alpha_{i}(g)$ for $i=1,2$ and all $g \in G$. Then the surfaces $\left(X_{1}, \sigma_{1}\right)$ and $\left(X_{2}, \sigma_{2}\right)$ are considered equivariantly equivalent or equivariantly isomorphic if there exist a biholomorphic map $\varphi: X_{1} \rightarrow X_{2}$ and a group automorphism $\psi \in \operatorname{Aut}(G)$ such that

$$
\alpha_{2}(g) \varphi(x)=\varphi\left(\alpha_{1}(\psi(g)) x\right) \text { and } \sigma_{2}(\varphi(x))=\varphi\left(\sigma_{1}(x)\right) .
$$

for all $x \in X_{1}$ and all $g \in G$.

Two (rational) surfaces $Y_{1}$ and $Y_{2}$ with actions $\alpha_{i}: G \rightarrow \operatorname{Aut}\left(Y_{i}\right)$ of a finite group $G$ are considered equivariantly equivalent if there exist a biholomorphic map $\varphi: Y_{1} \rightarrow Y_{2}$ and a group automorphism $\psi \in \operatorname{Aut}(G)$ such that $\alpha_{2}(g) \varphi(y)=\varphi\left(\alpha_{1}(\psi(g)) y\right)$ for all $y \in Y_{1}$ and all $g \in G$.

Remark 3.3. If two K3-surfaces $\left(X_{1}, \sigma_{1}\right)$ and $\left(X_{2}, \sigma_{2}\right)$ are $G$-equivariantly equivalent, then the quotient surfaces $X_{i} / \sigma_{i}$ are equivariantly equivalent with respect to the induced action of $G$.

Conversely, let $Y$ be a rational surface with two actions of a finite group $G$ which are equivalent in the above sense and let $\varphi \in \operatorname{Aut}(Y)$ be the isomorphism identifying these two actions. We consider a smooth $G$-invariant curve $B$ linearly equivalent to $-2 K_{Y}$ and the K3-surfaces $X_{B}$ and $X_{\varphi(B)}$ obtained as double covers branched along $B$ and $\varphi(B)$ equipped with their respective antisymplectic covering involution. If all elements of the group $G$ can be lifted to symplectic transformations on $X_{B}$ and $X_{\varphi(B)}$, then the central degree two extensions $E$ of $G$ acting on $X_{B}, X_{\varphi(B)}$, respectively, split as $E=E_{\text {symp }} \times C_{2}$ with $E_{\text {symp }}=G$. In this case the group $G$ acts by symplectic transformations on $X_{B}$ and $X_{\varphi(B)}$ and since for each $g \in G \subset \operatorname{Aut}(Y)$ there is only one choice of symplectic lifting $\tilde{g} \in E$ the surfaces $X_{B}$ and $X_{\varphi(B)}$ are $G$-equivariantly equivalent in strong sense introduced above.

3.1. The group $L_{2}(7)$. Let $G \cong L_{2}(7)=\operatorname{PSL}\left(2, \mathbb{F}_{7}\right)=\mathrm{GL}_{3}\left(\mathbb{F}_{2}\right)$ be the finite simple group of order 168 acting on a K3-surface $X$. As $G$ is simple this action is effective and symplectic. Let $\sigma$ be an antisymplectic involution on $X$ centralizing $G$. Since $G$ has an element of order seven, which by Remark 2.1 has precisely three fixed points $p_{1}, p_{2}, p_{3}$ in $X$, and $\sigma$ acts on this set of three points, we know that $\operatorname{Fix}_{X}(\sigma) \neq \emptyset$. I.e., the assumption $\operatorname{Fix}_{X}(\sigma) \neq \emptyset$ in Theorems 2.10 and 3.1 is implicitly true for this particular group.

The classification result for the group $G=L_{2}(7)$ can be deduced from a description of K3-surfaces with $C_{3} \ltimes C_{7}$-symmetry obtained in [FH08], Theorem 2 (cf. also Chapter 5 in [Fra08], Theorem 5.5). Using the same arguments but restricting our consideration to the larger group $L_{2}(7)$ we present a simplified proof of the following proposition.

Proposition 3.4. Let $X$ be a K3-surface with a symplectic action of the group $L_{2}(7)$ centralized by an antisymplectic involution $\sigma$. Then $X$ is equivariantly isomorphic to either $C_{\text {Klein }}=\left\{x_{1}^{3} x_{2}+x_{2}^{3} x_{3}+x_{3}^{3} x_{1}+x_{4}^{4}=0\right\} \subset \mathbb{P}_{3}$ or the double cover of $\mathbb{P}_{2}$ branched along $\operatorname{Hess}\left(C_{\text {Klein }}\right)$.

We begin with a classification of the quotient surface $Y=X / \sigma$.

Lemma 3.5. The quotient surface $Y$ is either $\mathbb{P}_{2}$ or a Del Pezzo surface of degree 2.

Proof. We may apply Theorem 2.10 to conclude that $Y$ is a $G$-minimal Del Pezzo surface and denote by $d=\operatorname{deg}(Y)=K_{Y}^{2}$ its degree.

If $d=1$, then the anticanonical system $\left|-K_{Y}\right|$ is known to have precisely one base point. In particular, this point is fixed with respect to the full automorphism group. Since $G$ does not admit a two-dimensional 
representation, this yields a contradiction. As there is no injective homomorphism $G \hookrightarrow \operatorname{Aut}\left(\mathbb{P}_{1} \times \mathbb{P}_{1}\right)=$ $\left(\mathrm{PSL}_{2}(\mathbb{C}) \times \mathrm{PSL}_{2}(\mathbb{C})\right) \rtimes C_{2}$ and since the blow-up of $\mathbb{P}_{2}$ in one or two points admits an equivariant contraction map to either $\mathbb{P}_{2}$ or $\mathbb{P}_{1} \times \mathbb{P}_{1}$ and is therefore never $G$-minimal, it follows that $d \neq 7,8$.

For the remaining Del Pezzo surfaces we consider the action of $G$ on their configurations of (-1)-curves. Noting that the maximal subgroups of $G$ are $C_{3} \ltimes C_{7}$ and $S_{4}$ of index 8 and 7, respectively, we find that the $G$-orbit of a (-1)-curve $E \subset Y$ consists of 7, 8, 14, 21, 24, 28 or more curves. Since the number of (-1)-curves on a Del Pezzo surface $Y$ of degree 3, 5, 6 equals 27, 10, 6, respectively, it follows that $d \notin\{3,5,6\}$. If $d=4$, then the union of (-1)-curves on $Y$ consists of two $G$-orbits of length 8. In particular, $\operatorname{Stab}_{G}(E) \cong C_{3} \ltimes C_{7}$ for any (-1)-curve $E \subset Y$. Blowing down $E$ to a point $p \in Y^{\prime}$ induces an action of $C_{3} \ltimes C_{7}$ on $Y^{\prime}$ fixing $p$. Since $C_{3} \ltimes C_{7}$ does not admit a two-dimensional representation, it follows that the normal subgroup $C_{7}$ acts trivially on $Y^{\prime}$ and therefore on $Y$. This is a contradiction and completes the proof of the lemma.

It remains to classify the branch locus $B$ in both cases.

Double covers of $\mathbb{P}_{2}$. The action of $L_{2}(7)$ on $\mathbb{P}_{2}$ is necessarily given by a three-dimensional represention. This follows from the fact that the group $L_{2}(7)$ does not admit nontrivial degree three central extensions. This can be derived from the cohomology group $H^{2}\left(L_{2}(7), \mathbb{C}^{*}\right) \cong C_{2}$ known as the Schur Multiplier. There are two isomorphism classes of three-dimensional representations and these differ by an outer automorphism. We may therefore consider one particular representation and check explicitly that in appropriately chosen coordinates the curve $B=\operatorname{Hess}\left(C_{\text {Klein }}\right)=\left\{x_{1}^{5} x_{2}+x_{3}^{5} x_{1}+x_{2}^{5} x_{3}-5 x_{1}^{2} x_{2}^{2} x_{3}^{2}=0\right\}$ is $L_{2}(7)$-invariant. As the notation indicates this sextic is the Hessian curve associated to Klein's quartic curve $C_{\text {Klein }}=\left\{x_{1}^{3} x_{2}+x_{2}^{3} x_{3}+x_{3}^{3} x_{1}=0\right\}$

In order to show that $B$ is the unique $G$-invariant sextic in $\mathbb{P}_{2}$ we consider the action of $G$ on $B$. The maximal possible isotropy group is $C_{7}$ and each $G$-orbit in $B$ consists of at least 21 elements. If there was another $G$-invariant smooth sextic curve $C \subset \mathbb{P}_{2}$, then the invariant set $B \cap C$ would consist of at most 36 points. This is a contradiction.

Let $X$ be the K3-surface obtained as a double cover of $\mathbb{P}_{2}$ branched along Hess $\left(C_{\text {Klein }}\right)$. It remains to check that $G$ lifts to a subgroup of $\operatorname{Aut}(X)$ : On $X$ we find an action of a central degree two extension $E$ of $L_{2}(7)$. Since $L_{2}(7)$ is simple, it follows that $E_{\text {symp }} \triangleleft E$ is mapped onto $L_{2}(7)$ and $E_{\text {symp }} \cong L_{2}(7)$. In particular, the group $E$ splits as $E_{\text {symp }} \times C_{2}$ where $C_{2}$ is generated by the antisymplectic covering involution. We have shown:

Proposition 3.6. Let $X$ be a K3-surface with a symplectic action of the group $L_{2}(7)$ centralized by an antisymplectic involution $\sigma$. If $Y=X / \sigma \cong \mathbb{P}_{2}$ then $X$ is equivariantly isomorphic to the double cover of $\mathbb{P}_{2}$ branched along $\operatorname{Hess}\left(C_{\text {Klein }}\right)$.

Double covers of Del Pezzo surfaces of degree two. Here we assume that the degree of $Y$ equals two and consider the anticanonical map $Y \rightarrow \mathbb{P}_{2}$. It has degree two, is branched along a smooth curve of degree four, and equivariant with respect to $\operatorname{Aut}(Y)$. We obtain an action of $G$ on $\mathbb{P}_{2}$ stabilizing a smooth quartic. Choosing one particular 3-dimensional representation of $G$ one checks by direct computation that the smooth curve $C_{\text {Klein }}=\left\{x_{1}^{3} x_{2}+x_{2}^{3} x_{3}+x_{3}^{3} x_{1}=0\right\}$ is invariant. It follows from classical invariant theory or from the intersection number argument applied above that $C_{\text {Klein }}$ is the unique quartic curve in $\mathbb{P}_{2}$ with this property. The curve $C_{\text {Klein }}$ is a prominent example since its automorphism group $\operatorname{Aut}\left(C_{\text {Klein }}\right)=G$ attains the maximal possible order $168=84\left(g\left(C_{\text {Klein }}\right)-1\right)$ allowed by the Hurwitz formula.

Consider the branch curve $B \subset Y$ of the covering $\pi: X \rightarrow Y$ and the preimage $C \subset Y$ of $C_{\text {Klein }}$. By construction, both curves belong to the linear system $\left|-2 K_{Y}\right|$. If they do not coincide, they meet in $\left(-2 K_{Y}\right)^{2}=8$ or less points. This contradicts the fact that the set $B \cap C$ must be $G$-invariant and therefore consists of at least 21 points in a $G$-orbit. 
It follows that the surface $X$ is a cyclic degree four cover of $\mathbb{P}_{2}$ branched along $C_{\text {Klein }}$ and can equivalently be described as the quartic hypersurface $\left\{x_{1}^{3} x_{2}+x_{2}^{3} x_{3}+x_{3}^{3} x_{1}+x_{4}^{4}=0\right\} \subset \mathbb{P}_{3}$. On $X$ there is action of the group $\tilde{G}=G \times C_{4}$ where $\tilde{G}_{\text {symp }}=G$. We have shown:

Proposition 3.7. Let $X$ be a K3-surface with a symplectic action of the group $L_{2}(7)$ centralized by an antisymplectic involution $\sigma$. If $Y=X / \sigma$ is a Del Pezzo surface of degree two then $X$ is equivariantly isomorphic to the cyclic degree 4 cover of $\mathbb{P}_{2}$ branched along $C_{\text {Klein }}$, i.e., Mukai's $L_{2}(7)$ example $\left\{x_{1}^{3} x_{2}+x_{2}^{3} x_{3}+x_{3}^{3} x_{1}+x_{4}^{4}=\right.$ $0\} \subset \mathbb{P}_{3}$.

Combining this with the previous proposition yields the classification result summarized in Proposition 3.4

Remark 3.8. Proposition 3.4 yields an alternative proof of the Main Theorem in [OZ02] classifying K3surfaces with actions of finite groups containing $G=L_{2}(7)$ as a subgroup of index four (cf. Corollary 1.3 in [FH08] and Theorem 6.1 and Lemma 6.2 in [Fra08])

Remark 3.9. In [FH08] K3-surfaces with a symplectic action of $C_{3} \ltimes C_{7}$ centralized by an antisymplectic involution are described as double cover of $\mathbb{P}_{2}$ branched along sextic curves in a 1-dimensional family $\mathscr{M}$. Up to equivalence, there is a unique singular curve $C_{\text {sing }}$ in this family. The K3-surface obtained as the minimal desingularization of the corresponding double cover of $\mathbb{P}_{2}$ is, by contruction, a double cover of the blow-up $b: Y \rightarrow \mathbb{P}_{2}$ at the seven singular points of $C_{\text {sing. }}$. The surface $Y$ is a Del Pezzo surface of degree two, its anticanonical map $\varphi: Y \rightarrow \mathbb{P}_{2}$ is branched along the proper transform $B$ of $C_{\text {sing }}$ in $Y$ and its image $\varphi(B)$ is isomorphic to Klein's quartic curve. It follows that the surface $\left\{x_{1}^{3} x_{2}+x_{2}^{3} x_{3}+x_{3}^{3} x_{1}+x_{4}^{4}=0\right\} \subset \mathbb{P}_{3}$ can also be characterized by the degeneration of the family $\mathscr{M}$ (cf. Theorem 1 in [FH08] and Theorem 5.4 in [Fra08]). The blow-up map $Y \rightarrow \mathbb{P}_{2}$ corresponds to the equivariant Mori reduction of $Y$ with respect to the subgroup $C_{3} \ltimes C_{7}$ of $G$.

3.2. The group $A_{6}$. Let $G \cong A_{6}$ be the alternating group of degree 6 acting on a K3-surface $X$. Since $A_{6}$ is a simple group this action effective and symplectic. Let $\sigma$ be an antisymplectic involution on $X$ centralizing $G$ and assume that $\operatorname{Fix}_{X}(\sigma) \neq \emptyset$. By Theorem 2.10, the K3-surface $X$ is a double cover of a Del Pezzo surface $Y$ with an effective action of $A_{6}$.

Lemma 3.10. The Del Pezzo surface $Y$ is isomorphic to $\mathbb{P}_{2}$ with a uniquely determined action of $A_{6}$ given by the nontrivial central extension $V=3$. $A_{6}$ of degree three known as Valentiner's group.

Proof. We go through the list of Del Pezzo surfaces according to their degree.

If $Y$ has degree one, then $\left|-K_{Y}\right|$ has precisely one base point which would have to be an $A_{6}$-fixed point. This is contrary to the fact that $A_{6}$ has no faithful two-dimensional representation.

We recall that the stabilizer of a (-1)-curve $E$ in $Y$ is either cyclic or dihedral (Remark 2.11). In particular, its order is at most 12 and therefore its index in $A_{6}$ is at least 30. This argument excludes Del Pezzo surfaces $Y$ of degree $\operatorname{deg}(Y) \in\{3,4,5,6,7\}$ since the number of $(-1)$-curves on $Y$ equals 27, 16, 10, 6, 3, respectively. Furthermore, the configuration of 56 exceptional curves on a Del Pezzo surface of degree 2 can neither be a single $A_{6}$-orbit nor the union of orbits and therefore $\operatorname{deg}(Y) \neq 2$.

As was noted before the blow-up of $\mathbb{P}_{2}$ in one point is never $G$-minimal, hence it remains to exclude $Y \cong \mathbb{P}_{1} \times \mathbb{P}_{1}$. Assume there is an action of $A_{6}$ on $\mathbb{P}_{1} \times \mathbb{P}_{1}$. Since $A_{6}$ has no subgroups of index two, it follows that $A_{6}<\mathrm{PSL}_{2}(\mathbb{C}) \times \mathrm{PSL}_{2}(\mathbb{C})$ and both canonical projections are $A_{6}$-equivariant. This yields a contradiction since $A_{6}$ admits neither an effective action on $\mathbb{P}_{1}$ nor nontrivial normal subgroups of ineffectivity.

It follows that $Y \cong \mathbb{P}_{2}$. The action of $A_{6}$ on $\mathbb{P}_{2}$ is given by linear representation of a degree three central extension of $A_{6}$. Since $A_{6}$ has no faithful three-dimensional representation, this extension is nontrivial and isomorphic the unique nontrivial degree three extension $V=3 . A_{6}$ known as Valentiner's group. Up to equivariant equivalence, there is a unique action of $A_{6}$ on $\mathbb{P}_{2}$. This follows from the classification of finite subgroup of $\mathrm{SL}_{3}(\mathbb{C})$ (cf. [MBD16], [Bli17], and [YY93]) and can also be derived as follows: 
We need to show that any two actions induced by projective representations $\rho_{i}$ of $A_{6}$ are equivalent. Restricting $\rho_{i}$ to the subgroup $A_{5}$ we obtain linear representation of $A_{5}$ and after a change of coordinates $\rho_{1}\left(A_{5}\right)=\rho_{2}\left(A_{5}\right) \subset \mathrm{SL}_{3}(\mathbb{C})$. We fix a subgroup $A_{4}$ in $A_{5}$ and consider its normalizer $N$ in $A_{6}$. The groups $N$ and $A_{5}$ generate the full group $A_{6}$ and it suffices to prove that $\rho_{1}(N)=\rho_{2}(N)$. This is shown by considering an explicit three-dimensional representation of $A_{4}<A_{5}$ and the normalizer $\mathscr{N}$ of $A_{4}$ inside $\mathrm{PSL}_{3}(\mathbb{C})$. The group $A_{4}$ has index two in $\mathscr{N}$ and therefore $\mathscr{N}=\rho_{1}(N)=\rho_{2}(N)$..

The covering $X \rightarrow Y$ is branched along an invariant curve $B$ of degree six. This curve is defined by an invariant polynomial $F_{A_{6}}$ of degree six, which is unique by Molien's formula. Its explicit equation is derived in [Cra99]. In appropriately chosen coordinates,

$$
F_{A_{6}}\left(x_{1}, x_{2}, x_{3}\right)=10 x_{1}^{3} x_{2}^{3}+9 x_{1}^{5} x_{3}+9 x_{2}^{3} x_{3}^{3}-45 x_{1}^{2} x_{2}^{2} x_{3}^{2}-135 x_{1} x_{2} x_{3}^{4}+27 x_{3}^{6} \text {. }
$$

The action of $A_{6}$ on $\mathbb{P}_{2}$ induces an action of a central degree two extension of $E$ on the double cover branched along $\left\{F_{A_{6}}=0\right\}$. By the same arguments as in the case $G=L_{2}(7)$ we may conclude that $E$ splits as $E_{\text {symp }} \times C_{2}=A_{6} \times C_{2}$ where $C_{2}$ is generated by the antisymplectic covering involution. This proves the existence of a unique K3-surface with $A_{6} \times C_{2}$-symmetry to which we refer as the Valentiner surface. We have shown:

Proposition 3.11. Let $X$ be a K3-surface with a symplectic action of $A_{6}$ and let $\sigma$ be an antisymplectic involution on $X$ centralizing $A_{6}$ with $\operatorname{Fix}_{X}(\sigma) \neq \emptyset$. Then $X$ is equivariantly isomorphic to the Valentiner surface.

3.3. The group $S_{5}$. Let $X$ be a K3-surface with a symplectic action of $G=S_{5}$ and let $\sigma$ denote an antisymplectic involution centralizing $G$. We assume that $\operatorname{Fix}_{X}(\sigma) \neq \emptyset$. We may apply Theorem 2.10 yielding that $X / \sigma=Y$ is a $G$-minimal Del Pezzo surface and $\pi: X \rightarrow Y$ is branched along a smooth connected curve $B$ of genus $g(B)=13-e(Y)$. We show that only very few Del Pezzo surfaces admit an effective action of $S_{5}$ or a smooth $S_{5}$-invariant curve of appropriate genus.

Lemma 3.12. The degree $\operatorname{deg}(Y)=d$ of the Del Pezzo surface $Y$ is either three or five.

Proof. We go through the list of $G$-minimal Del Pezzo surfaces and exclude all possible cases except $d \in$ $\{3,5\}$.

Assume $Y \cong \mathbb{P}_{2}$, i.e., $G \hookrightarrow \mathrm{PSL}_{3}(\mathbb{C})$, and let $\tilde{G}$ denote the preimage of $G$ in $\mathrm{SL}_{3}(\mathbb{C})$. Since $A_{5}$ has no nontrivial central extension of degree three, it follows that the preimage of $A_{5}<S_{5}$ in $\tilde{G}$ splits as $\tilde{A}_{5}=$ $A_{5} \times C_{3}$. Let $g \in S_{5}$ be any transposition and pick $\tilde{g}$ in its preimage with $\tilde{g}^{2}=$ id. Now $\tilde{g}$ and $A_{5}$ generate a copy of $S_{5}$ in $\mathrm{SL}_{3}(\mathbb{C})$. This is a contradiction since the irreducible representations of $S_{5}$ have dimensions $1,4,5$ or 6

Next assume that $Y$ is isomorphic to $\mathbb{P}_{1} \times \mathbb{P}_{1}$. We investigate the action of $S_{5}=A_{5} \rtimes C_{2}$ using the fact that $A_{5}$ is a simple group. Recalling $\operatorname{Aut}(Y)=\left(\mathrm{PSL}_{2}(\mathbb{C}) \times \mathrm{PSL}_{2}(\mathbb{C})\right) \rtimes C_{2}$ it follows that $A_{5}<\mathrm{PSL}_{2}(\mathbb{C}) \times \mathrm{PSL}_{2}(\mathbb{C})$, i.e, the canonical projections onto the factors are $A_{5}$-equivariant. If $A_{5}$ acts trivially on one of the factors, then it must act nontrivially on the second factor and in particular the generator $\tau$ of the outer $C_{2}$ in $S_{5}$ stabilizes the factors. It follows that $S_{5}$ acts effectively on the second factor. This is impossible since there is no effective action of $S_{5}$ on $\mathbb{P}_{1}$. We conclude that $A_{5}$ acts effectively on both factors and $\tau$ exchanges them. We consider an element $\lambda$ of order five in $A_{5}$ and chose coordinates on $\mathbb{P}_{1} \times \mathbb{P}_{1}$ such that $\lambda$ acts by $\left(\left[z_{1}: z_{2}\right],\left[w_{1}: w_{2}\right]\right) \mapsto\left(\left[\xi_{z_{1}}: z_{2}\right],\left[\xi^{a} w_{1}: w_{2}\right]\right)$ for some $a \in\{1,2,3,4\}$ and $\xi^{5}=1$. The automorphism $\lambda$ has four fixed points in $Y$. Since it lifts to a symplectic automorphism on the K3-surface $X$ with four fixed points, all fixed points must lie on the branch curve. The branch curve $B \subset Y$ is a smooth invariant curve linearly equivalent to $-2 K_{Y}$ and is therefore given by an $S_{5}$-semi-invariant polynomial $f$ of bidegree $(4,4)$. Since $f$ must be invariant with respect to the commutator subgroup $S_{5}^{\prime}=A_{5}$, it is a linear combination of $\lambda$-invariant monomials of bidegree $(4,4)$. For each choice of $a$ one lists all $\lambda$-invariant monomials of bidegree $(4,4)$. In the case $a=1$ these are $z_{1} z_{2}^{3} w_{1}^{4}, z_{1}^{2} z_{2}^{2} w_{1}^{3} w_{2}, z_{1}^{3} z_{2} w_{1}^{2} w_{2}^{2}, z_{1}^{4} w_{1} w_{2}^{3}, z_{2}^{4} w_{2}^{4}$. As $f$ must 
vanish at $p_{1} \ldots p_{4}$, one sees that it may not contain $z_{2}^{4} w_{2}^{4}$. The remaining monomials have a common component $z_{1} w_{1}$ such that $f$ factorizes and $B$ must be reducible, a contradiction. The same argument can be carried out for each choice of $a$. It follows that the action of $S_{5}$ on $\mathbb{P}_{1} \times \mathbb{P}_{1}$ does not admit invariant irreducible curves of bidegree $(4,4)$. This eliminates the case $Y \cong \mathbb{P}_{1} \times \mathbb{P}_{1}$.

Again using the fact that the largest subgroup of $S_{5}$ which can stabilize a (-1)-curve in $Y$ is the group $D_{12}$ of index 10, it follows that the number of (-1)-curves in a $G$-orbit is at least 10 and Del Pezzo surfaces of degree four or six can be excluded by considering the action of $G$ on their set of (-1)-curves.

If $d=2$, then the anticanonical map defines an $\operatorname{Aut}(Y)$-equivariant double cover of $\mathbb{P}_{2}$. The induced action of $S_{5}$ on $\mathbb{P}_{2}$ would have to be effective and therefore we obtain a contradiction as in the case $Y \cong \mathbb{P}_{2}$.

If $d=1$ then the anticanonical system $\left|-K_{Y}\right|$ is known to have precisely one base point which has to be fixed point of the action of $S_{5}$. Since $S_{5}$ has no faithful two-dimensional representation, this is a contradiction.

Double covers of Del Pezzo surfaces of degree three. The following example of a K3-surface $X$ with a symplectic action of $S_{5}$ can be found in Mukai's list [Muk88]. We identify an antisymplectic involution $\sigma$ on $X$ centralizing $S_{5}$ such that $X / \sigma$ is a Del Pezzo surface of degree three.

Example 3.13. Let $X$ be the K3-surface in $\mathbb{P}_{5}$ given by $\sum_{i=1}^{5} x_{i}=\sum_{i=1}^{6} x_{1}^{2}=\sum_{i=1}^{5} x_{i}^{3}=0$ and let $S_{5}$ act on $\mathbb{P}_{5}$ by permuting the first five variables and by the character sgn on the last variable. This induces a symplectic action of $S_{5}$ action on $X$ (cf. [Muk88]). The involution $\sigma: \mathbb{P}_{5} \rightarrow \mathbb{P}_{5}$ given by $\left[x_{1}: x_{2}: x_{3}: x_{4}\right.$ : $\left.x_{5}: x_{6}\right] \mapsto\left[x_{1}: x_{2}: x_{3}: x_{4}: x_{5}:-x_{6}\right]$ defines an antisymplectic involution on $X$ centralizing the action of $S_{5}$. The quotient $Y$ of $X$ by $\sigma$ is given by restricting then rational map $\left[x_{1}: x_{2}: x_{3}: x_{4}: x_{5}: x_{6}\right] \mapsto$ $\left[x_{1}: x_{2}: x_{3}: x_{4}: x_{5}\right]$ to $X$. The surface $Y$ is given by $\left\{\sum_{i=1}^{5} y_{i}=\sum_{i=1}^{5} y_{i}^{3}=0\right\} \subset \mathbb{P}_{4}$ and is isomorphic to the Clebsch diagonal surface $\left\{z_{1}^{2} z_{2}+z_{1} z_{3}^{2}+z_{3} z_{4}^{2}+z_{4} z_{2}^{2}=0\right\} \subset \mathbb{P}_{3}$ (cf. Theorem 10.3.10 in [Dol09]), a Del Pezzo surface of degree three. The branch set $B$ is given by $\left\{\sum_{i=1}^{5} y_{1}^{2}=0\right\} \cap Y \subset \mathbb{P}_{4}$. By the following proposition, this example is the unique K3-surface with $S_{5} \times\langle\sigma\rangle$-symmetry such that $X / \sigma$ is a Del Pezzo surface of degree three.

Proposition 3.14. Let $X$ be a K3-surface with a symplectic action of the group $S_{5}$ centralized by an antisymplectic involution $\sigma$. If $Y=X / \sigma$ is a Del Pezzo surface of degree three, then $X$ is equivariantly isomorphic to Mukai's $S_{5}$-example $\left\{\sum_{i=1}^{5} x_{i}=\sum_{i=1}^{6} x_{1}^{2}=\sum_{i=1}^{5} x_{i}^{3}=0\right\} \subset \mathbb{P}_{5}$.

Proof. We consider the Aut $(Y)$-equivariant embedding of the degree three Del Pezzo surface $Y$ into $\mathbb{P}_{3}$ given by the anticanonical map. Any automorphism of $Y$ is induced by an automorphism of the ambient projective space. It follows from the representation and invariant theory of the group $S_{5}$ that a Del Pezzo surface of degree three with an effective action of the group $S_{5}$ is equivariantly isomorphic the Clebsch cubic $\left\{z_{1}^{2} z_{2}+z_{1} z_{3}^{2}+z_{3} z_{4}^{2}+z_{4} z_{2}^{2}=0\right\} \subset \mathbb{P}_{3}$ (cf. Theorems 10.3.9 and 10.3.10, Table 10.3 in [Dol09]). We show that the ramification curve $B \in\left|-2 K_{Y}\right|$ is given by intersecting $Y$ with a quadric in $\mathbb{P}_{3}$.

Applying the formula $h^{0}\left(Y, \mathscr{O}\left(-r K_{Y}\right)\right)=1+\frac{1}{2} r(r+1) \cdot \operatorname{deg}(Y)$ (cf. e.g. Lemma 8.3.1 in [Dol09]) we obtain $h^{0}\left(Y, \mathscr{O}\left(-2 K_{Y}\right)\right)=10$. This is also the dimension of the space of sections of $\mathscr{O}(2)$ in $\mathbb{P}_{3}$. It follows that the restriction map $H^{0}\left(\mathbb{P}_{3}, \mathscr{O}(2)\right) \rightarrow H^{0}\left(Y, \mathscr{O}\left(-2 K_{Y}\right)\right)$ is surjective and $B=Y \cap Q$ for some quadric $Q=\{f=0\}$ in $\mathbb{P}_{3}$.

Since $B$ is an $S_{5}$-invariant curve in $Y$, it follows that for each $g \in S_{5}$ the intersection of $g Q=\left\{f \circ g^{-1}=0\right\}$ and $Y$ coincides with $B$. It follows that there exists a constant $c \in \mathbb{C}^{*}$ such that $\left(f \circ g^{-1}\right)-c f$ vanishes identically on $Y$. Since $Y$ is irreducible, this implies $f \circ g^{-1}=c f$. It follows that the polynomial $f$ is an $S_{5}$-semi-invariant and therefore invariant with respect to the commutator subgroup $A_{5}$.

We have previously noted that after a suitable linear change of coordinates the surface $Y$ is given by $\left\{\sum_{i=1}^{5} y_{i}=\sum_{i=1}^{5} y_{i}^{3}=0\right\} \subset \mathbb{P}_{4}$ where $S_{5}$ acts by permutation. In these coordinates the semi-invariant 
polynomial $f$ is given by $a \sum_{i=1}^{5} y_{i}^{2}+b\left(\sum_{i=1}^{5} y_{i}\right)^{2}=0$ for some $a, b \in \mathbb{C}$. In particular, $B=Y \cap\{f=0\}=$ $Y \cap\left\{\sum_{i=1}^{5} y_{i}^{2}=0\right\}$ and $X$ is Mukai's $S_{5}$-example discussed in Example 3.13 ,

Double covers of Del Pezzo surfaces of degree five. A second class of candidates of K3-surfaces with $S_{5} \times C_{2}$-symmetry is given by double covers of Del Pezzo surfaces of degree five.

Remark 3.15. Any two Del Pezzo surfaces of degree five are isomorphic and the automorphisms group of a Del Pezzo surface $Y$ of degree five is $S_{5}$. The ten (-1)-curves on $Y$ form a graph known as the Petersen graph. This graph has $S_{5}$-symmetry and every symmetry of the abstract graph is induced by a unique automorphism of the Del Pezzo surface.

Proposition 3.16. Let $X$ be a K3-surface with a symplectic action of the group $S_{5}$ centralized by an antisymplectic involution $\sigma$. If $Y=X / \sigma$ is a Del Pezzo surface of degree five, then $X$ is equivariantly isomorphic to the minimal desingularization of the double cover of $\mathbb{P}_{2}$ branched along the sextic

$$
\begin{aligned}
C_{S_{5}}=\{ & \left\{x_{1}^{4} x_{2} x_{3}+2 x_{1} x_{2}^{4} x_{3}+2 x_{1} x_{2} x_{3}^{4}-2 x_{1}^{4} x_{2}^{2}-2 x_{1}^{4} x_{3}^{2}-2 x_{1}^{2} x_{2}^{4}-2 x_{1}^{2} x_{3}^{4}\right. \\
& -2 x_{2}^{4} x_{3}^{2}-2 x_{2}^{2} x_{3}^{4}+2 x_{1}^{3} x_{2}^{3}+2 x_{1}^{3} x_{3}^{3}+2 x_{2}^{3} x_{3}^{3}+x_{1}^{3} x_{2}^{2} x_{3}+x_{1}^{3} x_{2} x_{3}^{2} \\
& \left.+x_{1}^{2} x_{2}^{3} x_{3}+x_{1}^{2} x_{2} x_{3}^{3}+x_{1} x_{2}^{3} x_{3}^{2}+x_{1} x_{2}^{2} x_{3}^{3}-6 x_{1}^{2} x_{2}^{2} x_{3}^{2}=0\right\} .
\end{aligned}
$$

Proof. Let $B \subset Y$ denote the branch locus of the covering $X \rightarrow Y$. The curve $B$ is invariant with respect to the full automorphism group of $Y$. The Del Pezzo surface $Y$ is the blow-up $b: Y \rightarrow \mathbb{P}_{2}$ of four points $p_{1}, p_{2}, p_{3}, p_{4} \in \mathbb{P}_{2}$ in general position. We may choose coordinates $\left[x_{1}: x_{2}: x_{3}\right]$ on $\mathbb{P}_{2}$ such that $p_{1}=[1$ : $0: 0], p_{2}=[0: 1: 0], p_{3}=[0: 0: 1], p_{4}=[1: 1: 1]$, and consider the $S_{4}$-action on $\mathbb{P}_{2}$ permuting the points $\left\{p_{i}\right\}$. The isotropy at each point $p_{i}$ is isomorphic to $S_{3}$ and induces an effective $S_{3}$-action on $E_{i}=b^{-1}\left(p_{i}\right)$.

Let $E$ be any (-1)-curve on $Y$. By adjunction $E \cdot B=2$. Since $Y$ contains precisely ten (-1)-curves forming an $S_{5}$-orbit, the group $H=\operatorname{Stab}_{S_{5}}(E)$ has order 12 and all stabilizer groups of (-1)-curves in $Y$ are conjugate. It follows that the group $H$ contains $S_{3}$, which is acting effectively on $E$, and therefore $H$ is isomorphic to the dihedral group of order 12. The points of intersection $B \cap E$ form an $H$-invariant subset of $E$. Since $H$ has no fixed points in $E$ and precisely one orbit $H . p=\{p, q\}$ consisting of two elements, it follows that $B$ meets $E$ transversally in $p$ and $q$. In particular, each curve $E_{i}$ meets $B$ in two points and the image $C=b(B)$ is a sextic curve with four nodes $\left\{p_{1}, \ldots, p_{4}\right\}$. It is invariant with respect to the action of $S_{4}$ given by permutation on $p_{1}, \ldots p_{4}$. For simplicity, we first only consider the action of $S_{3}$ permuting $p_{1}, p_{2}, p_{3}$ and conclude that $C$ is given by $\left\{f=\sum a_{i} f_{i}=0\right\}$ for

$$
\begin{array}{lrl}
f_{1}=x_{1}^{6}+x_{2}^{6}+x_{3}^{6}, & f_{5}=x_{1}^{3} x_{2}^{3}+x_{1}^{3} x_{3}^{3}+x_{2}^{3} x_{3}^{3}, \\
f_{2}=x_{1}^{5} x_{2}+x_{1}^{5} x_{3}+x_{1} x_{2}^{5}+x_{1} x_{3}^{5}+x_{2}^{5} x_{3}+x_{2} x_{3}^{6}, & f_{6}=x_{1}^{3} x_{2}^{2} x_{3}+x_{1}^{3} x_{2} x_{3}^{2}+x_{1}^{2} x_{2}^{3} x_{3} \\
f_{3}=x_{1}^{4} x_{2} x_{3}+x_{1} x_{2}^{4} x_{3}+x_{1} x_{2} x_{3}^{4}, & & +x_{1}^{2} x_{2} x_{3}^{3}+x_{1} x_{2}^{3} x_{3}^{2}+x_{1} x_{2}^{2} x_{3}^{3}, \\
f_{4}=x_{1}^{4} x_{2}^{2}+x_{1}^{4} x_{3}^{2}+x_{1}^{2} x_{2}^{4}+x_{1}^{2} x_{3}^{4}+x_{2}^{4} x_{3}^{2}+x_{2}^{2} x_{3}^{4}, & f_{7}=x_{1}^{2} x_{2}^{2} x_{3}^{2} .
\end{array}
$$

The fact that $C$ passes through $p_{i}$ and is singular at $p_{i}$ yields $a_{1}=a_{2}=0$ and $3 a_{3}+6 a_{4}+3 a_{5}+6 a_{6}+a_{7}=0$. The two tangent lines of $C$ at the node $p_{i}$ correspond to the unique $\operatorname{Stab}\left(E_{i}\right)$-orbit of length two in $E_{i}$. We consider the point $p_{3}$ and the subgroup $S_{3}<S_{4}$ stabilizing $p_{3}$. The action of $S_{3}$ on $E_{3}$ is given by the linearized $S_{3}$-action on the set of lines through $p_{3}$. One checks that in local affine coordinates $\left(x_{1}, x_{2}\right)$ the unique orbit of length two corresponds to the line pair $x_{1}^{2}-x_{1} x_{2}+x_{2}^{2}=0$. Dehomogenizing $f$ at $p_{3}$, i.e., setting $x_{3}=1$, we obtain the local equation $f_{\text {dehom }}$ of $C$ at $p_{3}$. The polynomial $f_{\text {dehom }}$ modulo terms of order three or higher must be a multiple of $x_{1}^{2}-x_{1} x_{2}+x_{2}^{2}=0$. Therefore $a_{3}=-a_{4}$.

Next we consider the intersection of $C$ with the line $L_{34}=\left\{x_{1}=x_{2}\right\}$ joining $p_{3}$ and $p_{4}$. We know that $\left.f\right|_{L_{34}}$ vanishes of order two at $p_{3}$ and $p_{4}$ and at one or two further points on $L_{34}$. Let $\widetilde{L}_{34}$ denote the proper transform of $L_{34}$ inside the Del Pezzo surface $Y$. The curve $\widetilde{L}_{34}$ is a (-1)-curve, hence its stabilizer 
$\operatorname{Stab}_{G}\left(\widetilde{L}_{34}\right)$ is isomorphic to $D_{12}=S_{3} \times C_{2}$. The factor $C_{2}$ acts trivially on $\widetilde{L}_{34}$. Since the intersection of $\widetilde{L}_{34}$ with $B$ is $\operatorname{Stab}_{G}\left(\widetilde{L}_{34}\right)$ invariant, it follows that $\widetilde{L}_{34} \cap B$ is the unique $S_{3}$-orbit a length two in $\widetilde{L}_{34}$. We wish to transfer our determination of the unique $S_{3}$-orbit of length two in $E_{3}$ above to the curve $\widetilde{L}_{34}$ using an automorphism of $Y$ mapping $E_{3}$ to $\widetilde{L}_{34}$. Consider the automorphism $\varphi$ of $Y$ induced by the birational map of $\mathbb{P}_{2}$ given by $\left[x_{1}: x_{2}: x_{3}\right] \mapsto\left[x_{1}\left(x_{3}-x_{2}\right): x_{3}\left(x_{1}-x_{2}\right): x_{1} x_{3}\right]$ (cf. Theorem 10.2.2 in [Dol09]) and let $\psi$ be the automorphism of $Y$ induced by the permutation of the points $p_{2}$ and $p_{3}$ in $\mathbb{P}_{2}$. Then $\psi \circ \varphi$ is an automorphism of $Y$ mapping $E_{3}$ to $\widetilde{L}_{34}$. If $\left[X_{1}: X_{2}\right]$ denote homogeneous coordinates on $E_{3}$ induced by the affine coordinates $\left(x_{1}, x_{2}\right)$ in a neighbourhood of $p_{3}$, then a point $\left[X_{1}: X_{2}\right] \in E_{3}$ is mapped to the point corresponding to $\left[X_{1}: X_{1}: X_{1}-X_{2}\right] \in L_{34} \subset \mathbb{P}_{2}$. It was derived above that the unique $S_{3}$-orbit of length two in $E_{3}$ is given by $X_{1}^{2}-X_{1} X_{2}+X_{2}^{2}$ and it follows that the unique $S_{3}$-orbit of length two in $\widetilde{L}_{34}$ corresponds to the points $\left[x_{1}: x_{1}: x_{3}\right] \in \mathbb{P}_{2}$ fulfilling $x_{1}^{2}-x_{1} x_{3}+x_{3}^{2}=0$. Therefore, $\left.f\right|_{L_{34}}$ is a multiple of polynomial given by $x_{1}^{2}\left(x_{1}-x_{3}\right)^{2}\left(x_{1}^{2}-x_{1} x_{3}+x_{3}^{2}\right)$. Comparing coefficients with $f\left(x_{1}: x_{1}: x_{3}\right)$ yields

$$
\begin{aligned}
2 a_{3}+2 a_{6} & =2 a_{5}+2 a_{6}, & 2 a_{4}+a_{5} & =2 a_{4}+a_{3}, \\
8 a_{4}+4 a_{5} & =2 a_{4}+2 a_{6}+a_{7}, & -6 a_{4}-3 a_{5} & =2 a_{5}+2 a_{6} .
\end{aligned}
$$

We conclude $a_{3}=a_{5}=-a_{4}=2, a_{6}=1$, and $a_{7}=-6$. So if $X$ as in the proposition exists, it is the double cover of $Y$ branched along the proper transform of $\left\{f=2 f_{3}-2 f_{4}+2 f_{5}+f_{6}-6 f_{7}=0\right\}$ in $Y$.

In order to prove existence, let $X$ be the minimal desingularization of the double cover of $\mathbb{P}_{2}$ branched along $\{f=0\}$. Then $X$ is the double cover of the Del Pezzo surface $Y$ of degree five branched along the proper transform $D$ of $\{f=0\}$ in $Y$. Since all automorphisms of $Y$ are induced by explicit biholomorphic or birational transformation of $\mathbb{P}_{2}$ one can check by direct computations that $D$ is in fact invariant with respect to the action of $\operatorname{Aut}(Y)=S_{5}$.

On $X$ there is an action of a central extension $E$ of $S_{5}$, \{id $\} \rightarrow C_{2} \rightarrow E \rightarrow S_{5} \rightarrow\{$ id $\}$. Let $E_{\text {symp }}$ be the subgroup of symplectic automorphisms in $E$. Since $E$ contains the antisymplectic covering involution $E_{\text {symp }} \neq E$. The image $N$ of $E_{\text {symp }}$ in $S_{5}$ is normal and therefore either $N \cong S_{5}$ or $N \cong A_{5}$. If $N \cong A_{5}$ and $\left|E_{\text {symp }}\right|=60$, then $E_{\text {symp }} \cong A_{5}$. Lifting any transposition from $S_{5}$ to an element $g$ of order two in $E$, the group generated by $g$ and $E_{\text {symp }}$ inside $E$ is isomorphic to $S_{5}$. It follows that $E$ splits as $S_{5} \times C_{2}$ and $E / E_{\text {symp }} \cong C_{2} \times C_{2}$. Since this is not cyclic, we obtain a contradiction. If $N \cong A_{5}$ and $\left|E_{\text {symp }}\right|=120$, then $E=E_{\text {symp }} \times C_{2}$, where the outer $C_{2}$ is generated by the antisymplectic covering involution $\sigma$, and $E / C_{2}=S_{5}$ implies that $E_{\text {symp }} \cong S_{5}$. This is contradictory to the assumption $N \cong A_{5}$. In the last remaining case $N \cong S_{5}$. Since $E_{\text {symp }} \neq E$, also $E_{\text {symp }} \cong S_{5}$ and $E$ splits as $E_{\text {symp }} \times C_{2}$. It follows that the action of $S_{5}$ on $Y$ induces an symplectic action of $S_{5}$ on the double cover $X$ centralized by the antisymplectic covering involution. This completes the proof of the proposition.

We summarize Propositions 3.14 and 3.16 as follows.

Proposition 3.17. Let $X$ be a K3-surface with a symplectic action of the group $S_{5}$ centralized by an antisymplectic involution $\sigma$ with $\operatorname{Fix}_{X}(\sigma) \neq \emptyset$. Then $X$ is equivariantly isomorphic to either Mukai's $S_{5}$-example or the minimal desingularization of the double cover of $\mathbb{P}_{2}$ branched along the sextic $C_{S_{5}}$.

3.4. The group $M_{20}=C_{2}^{4} \rtimes A_{5}$. We assume that a K3-surface $X$ with a symplectic action of $M_{20}$ centralized by an antisymplectic involution $\sigma$ and $\operatorname{Fix}_{X}(\sigma) \neq \emptyset$ exists. Applying Theorem 2.10 we see that $X \rightarrow Y$ is branched along a single $M_{20}$-invariant smooth curve $B$ with $g(B) \geq 3$ on the Del Pezzo surface $Y$. By Hurwitz's formula, $|\operatorname{Aut}(B)| \leq 84(g(B)-1)$, the genus of $B$ must be at least twelve. As $B$ is linearly equivalent to $-2 K_{Y}$, the adjunction formula $2 g(B)-2=\left(K_{Y}, B\right)+B^{2}=2 K_{Y}^{2}$ implies $\operatorname{deg}(Y)=K_{Y}^{2} \geq 11$. Since the degree of a Del Pezzo surface is at most nine this is a contradiction yielding the following nonexistence result.

Proposition 3.18. There does not exist a K3-surface with a symplectic action of $M_{20}$ centralized by an antisymplectic involution $\sigma$ with $\operatorname{Fix}_{X}(\sigma) \neq \emptyset$. 
3.5. The group $F_{384}=C_{2}^{4} \rtimes S_{4}$. Before we prove non-existence of K3-surfaces with $F_{384} \times C_{2}$-symmetry, we note the following useful fact about $S_{4}$-actions on Riemann surfaces.

Remark 3.19. The group $S_{4}$ does not admit an effective action on a Riemann surface of genus one or two. The first case can be excluded by using the explicit shape of the automorphism group of a Riemann surface $T$ of genus one, $\operatorname{Aut}(T)=L \ltimes T$ for $L \in\left\{C_{2}, C_{4}, C_{6}\right\}$. The second case is excluded by considering the quotient by the hyperelliptic involution branched at six points. Since $S_{4}$ has no normal subgroup of order two, the induced action of $S_{4}$ on the quotient $\mathbb{P}_{1}$ is effective and therefore has precisely one orbit consisting of six points. The isotropy group at the corresponding points in the hyperelliptic curve would be isomorphic to $C_{4} \times C_{2}$, which is contradiction.

Proposition 3.20. There does not exists a K3-surface with a symplectic action of $F_{384}$ centralized by an antisymplectic involution $\sigma$ with $\operatorname{Fix}_{X}(\sigma) \neq \emptyset$.

Proof. As above, assume that a K3-surface $X$ with these properties exists and apply Theorem 2.10 to see that $X \rightarrow Y$ is branched along a single $F_{384}$-invariant smooth curve $B$ on the Del Pezzo surface $Y$. By Hurwitz's formula the genus of $B$ is at least 6 . It now follows from adjunction, $K_{Y}^{2}=g(B)-1$, that the degree of the Del Pezzo surface $Y$ is at least five. We consider the action of $F_{384}$ on the configuration of (-1)-curves on $Y$ and recall that the order of a stabilizer of a (-1)-curve in $Y$ is at most twelve (cf. Remark 2.11) and therefore has index greater than or equal to 32 in $G$. It follows that $Y$ is either $\mathbb{P}_{1} \times \mathbb{P}_{1}$ or $\mathbb{P}_{2}$. In the first case, the canonical projections of $\mathbb{P}_{1} \times \mathbb{P}_{1}$ are equivariant with respect to a subgroup of index two in $F_{384}$ and thereby contradict Lemma 2.2. Consequently, $Y \cong \mathbb{P}_{2}$ and $g(B)=10$.

We use the realization of $F_{384}$ as a semi-direct product $C_{4}^{2} \rtimes S_{4}$ (cf. [Muk88]) and consider the quotient $Q$ of the branch curve $B$ by the normal subgroup $N=C_{4}^{2}$. On $Q$ there is the induced action of $S_{4}$. It follows from the remark above that $Q$ is either rational or $g(Q)>2$. In the second case the Riemann-Hurwitz formula applied to the covering $B \rightarrow Q$,

$$
-18=e(B)=16 e(Q)-\text { branch point contributions } \leq-64,
$$

yields a contradiction. It follows that $Q$ is a rational curve and that the branch point contribution of the covering $B \rightarrow Q$ is 50 . Since isotropy groups at points in $B$ must be cyclic, the only possible isotropy subgroups of $N=C_{4}^{2}$ at points in $B$ are $C_{2}$ and $C_{4}$ and have index four or eight. The full branch point contribution must therefore be a multiple of four. This contradiction yields the non-existence claimed.

3.6. The group $A_{4,4}=C_{2}^{4} \rtimes A_{3,3}$. By $S_{p, q}$ for $p+q=n$ we denote a subgroup $S_{p} \times S_{q}$ of $S_{n}$ preserving a partition of the set $\{1, \ldots, n\}$ into two subsets of cardinality $p$ and $q$. The intersection of $S_{p, q}$ with $A_{n}$ is denoted by $A_{p, q}$.

Proposition 3.21. There does not exists a K3-surface with a symplectic action of $A_{4,4}$ centralized by an antisymplectic involution $\sigma$ with $\operatorname{Fix}_{X}(\sigma) \neq \emptyset$.

Proof. We again assume that a K3-surface with these properties exists and applying Theorem 2.10 we see that $X \rightarrow Y$ is branched along a single $A_{4,4}$-invariant smooth curve $B$ on the Del Pezzo surface $Y$. The group $A_{4,4}$ is a semi-direct product $C_{2}^{4} \rtimes A_{3,3}$ (see e.g. [Muk88]). We consider the quotient $Q$ of $B$ by the normal subgroup $N \cong C_{2}^{4}$. On $Q$ there is an action of $A_{3,3}$. Since $A_{3,3}$ contains the subgroup $C_{3} \times C_{3}$, it follows that $Q$ not rational. In particular, $e(Q) \leq 0$. We apply the Riemann-Hurwitz formula to the covering $B \rightarrow Q$,

$$
2-2 g(B)=e(B)=16 e(Q)-\text { branch point contributions } \leq \text {-branch point contributions. }
$$

As above, isotropy groups must be cyclic and the maximal possible isotropy group of the $C_{2}^{4}$-action on $B$ is $C_{2}$, which has index eight in $C_{2}^{4}$. Consequently, the branch point contribution at each branch point is eight. The action of $C_{3} \times C_{3}<A_{3,3}$ on $Q$ has orbits of length greater than or equal to three. Therefore, the total branch point contribution must be greater than or equal to 24. In particular, $g(B)=\operatorname{deg}(Y)+1 \geq 13$ contrary to $\operatorname{deg}(Y) \leq 9$. 
3.7. The groups $T_{192}=\left(Q_{8} * Q_{8}\right) \rtimes S_{3}$ and $H_{192}=C_{2}^{4} \rtimes D_{12}$. By $Q_{8}$ we denote the quaternion group $\{ \pm 1, \pm I, \pm J, \pm K\}$ where $I^{2}=J^{2}=K^{2}=I J K=-1$. The central product $Q_{8} * Q_{8}$ is defined as the quotient of $Q_{8} \times Q_{8}$ by the central involution $(-1,-1)$. Most importantly, we benefit from the observation that both groups $T_{192}$ and $H_{192}$ are semi-direct products $C_{2}^{3} \rtimes S_{4}$ (cf. [Muk88]).

We prove the following non-existence result.

Proposition 3.22. For $G=T_{192}$ or $G=H_{192}$ there does not exists a K3-surface with a symplectic action of $G$ centralized by an antisymplectic involution $\sigma$ with $\operatorname{Fix}_{X}(\sigma) \neq \emptyset$.

Assume that a K3-surface as in the proposition exists. Applying Theorem 2.10 we see that $X \rightarrow Y$ is branched along a single $G$-invariant smooth curve $C$ on the Del Pezzo surface $Y$. The genus of $C$ is at least four by Hurwitz's formula and therefore $d=\operatorname{deg}(Y) \geq 3$.

The cases $3 \leq d \leq 7$. We consider the action of $G$ on the Del Pezzo surface $Y$ of degree $\geq 3$, in particular the induced action on its configuration of $(-1)$-curves. By Remark 2.11 the stabilizer of a (-1)-curve in $Y$ has index $\geq 16$ in $G$ and we may immediately exclude the cases $\operatorname{deg}(Y)=3,5,6,7$. The automorphism group of a Del Pezzo surface of degree four is $C_{2}^{4} \rtimes \Gamma$ for $\Gamma \in\left\{C_{2}, C_{4}, S_{3}, D_{10}\right\}$ (cf. Section 10.2.2 in [Dol09]). In particular, the maximal possible order is 160 and therefore $\operatorname{deg}(Y) \neq 4$. It remains to consider the cases $Y \cong \mathbb{P}_{2}$ and $Y \cong \mathbb{P}_{1} \times \mathbb{P}_{1}$

The case $Y \cong \mathbb{P}_{2}$. If $Y \cong \mathbb{P}_{2}$ than $g(C)=10$. We consider the quotient $Q$ of $C$ by the normal subgroup $N=C_{2}^{3}$ and obtain

$$
-18=e(C)=8 \cdot e(Q)-\text { branch point contributions. }
$$

By Lemma 3.19 the quotient $Q$ is either rational or $g(Q)>2$, i.e., $e(Q) \leq-4$. Since the second case is impossible it follows that $Q$ is a rational curve and the branch point contribution must be 34 . The maximal possible isotropy subgroup of $N=C_{2}^{3}$ at a point in $C$ is $C_{2}$ and the full branch point contribution must be divisible by four. This is a contradiction.

The case $Y \cong \mathbb{P}_{1} \times \mathbb{P}_{1}$. Assume that $Y \cong \mathbb{P}_{1} \times \mathbb{P}_{1}$. The canonical projection $\pi_{1,2}: Y \rightarrow \mathbb{P}_{1}$ is equivariant with respect to a subgroup $\tilde{G}$ of $G$ of index at most two. It follows that $\tilde{G}$ fits into the exact sequences $\{\mathrm{id}\} \rightarrow I_{i} \rightarrow \tilde{G} \stackrel{\left(\pi_{i}\right)_{*}}{\rightarrow} H_{i} \rightarrow\{\mathrm{id}\}$, where $I_{i} \cong C_{2} \times C_{2}$ is the ineffectivity of the induced $H$-action on the base $\pi_{i}(Y)$ and $H_{i} \cong S_{4}$ (cf. proof of Lemma 2.2). We consider the realization of $G$ as a semi-direct product $C_{2}^{3} \rtimes S_{4}=N \rtimes S_{4}$ and denote by $A=\tilde{G} \cap N$ the intersection of the normal subgroup $N$ with the index two normal subgroup $\tilde{G}$.

Lemma 3.23. The group $A$ is isomorphic to $C_{2} \times C_{2}$.

Proof. It is sufficient to exclude the case $A=N$. We argue by contradiction and assume $A=N$. The commutator subgroup of $S_{4}$ is $A_{4}$ and therefore $A_{4}<G^{\prime}<\tilde{G}<G$. Since $\tilde{G}$ is known to be of order 96 , it follows that $\tilde{G}=N \rtimes A_{4}$. We consider the isotropy group $I_{1} \cong C_{2} \times C_{2}<\tilde{G}$ and its intersection with $A=N$.

If $A \cap I_{1}=\{$ id $\}$, then $A=N \cong C_{2}^{3}$ acts effectively on $\pi_{1}(Y)=\mathbb{P}_{1}$, a contradiction. If $A \cap I_{1}=I_{1}$, i.e., if $I_{1}$ is contained in $A$, then the quotient group $\tilde{G} / I_{1}$, which acts effectively on $\mathbb{P}_{1}$ by definition, is isomorphic to $C_{2} \rtimes A_{4} \cong C_{2} \times A_{4}$. This group does however not admit an effective action on $\mathbb{P}_{1}$.

It remains to consider the case $A \cap I_{1} \cong C_{2}$. The only nontrivial normal subgroup of $A_{4}$ is isomorphic to $C_{2} \times C_{2}$. Since $I_{1} \cong C_{2} \times C_{2}$ is not contained in $A_{4}$ in the case under consideration, it follows that $I_{1} \cap A_{4}=\{\mathrm{id}\}$. Written as a subgroup of the semi-direct product $\tilde{G}=N \rtimes A_{4}$ the group $I_{1} \cong C_{2} \times C_{2}$ is of the form $I_{1}=\left\{(\mathrm{id}, \mathrm{id}),\left(\sigma_{1}, \mathrm{id}\right),\left(\sigma_{2}, a\right),\left(\sigma_{3}, a\right)\right\}$ for $\sigma_{i} \in N$ and $a \in A_{4}$. Using the fact that $I_{1}$ is a normal subgroup of $\tilde{G}$ one finds that $\mathrm{gag}^{-1}=a$ for each $g \in A_{4}<\tilde{G}$, a contradiction. 
By the lemma above, the set $N \backslash A$ consists of four elements. These are involutions in $G$ not respecting the product structure of $Y \cong \mathbb{P}_{1} \times \mathbb{P}_{1}$. In particular, each element of $N \backslash A$ exchanges the factors of $Y$. Since both $N$ and $A$ are normal subgroup of $G$, it follows that $G$ acts on $N, A$, and also $N \backslash A$ by conjugation. We consider an element $\lambda$ of order three contained in $A_{4}$, the commutator of the chosen copy of $S_{4}$ inside $G$. In particular, $\lambda \in A_{4}=S_{4}^{\prime}<G^{\prime}<\tilde{G}<G$. The action of $\lambda$ on the set $N \backslash A$ has at least one fixed point, i.e., there exists an element $\sigma \in N \backslash A$ such that $\lambda \sigma \lambda^{-1}=\sigma$.

After a suitable change of coordinates, the action of $\sigma$ on $Y$ is of the form $\sigma(z, w)=(w, z)$. Since $\lambda \in \tilde{G}$ respects the product structure and commutes with $\sigma$, it follows that $\lambda(z, w)=\left(\lambda_{z} z, \lambda_{z} w\right)$. for some $\lambda_{z} \in \mathrm{PSL}_{2}(\mathbb{C})$ of order three. We choose homogeneous coordinates on $\pi_{i}(Y) \cong \mathbb{P}_{1}$ such that $\lambda(z, w)=$ $\lambda\left(\left[z_{0}: z_{1}\right],\left[w_{0}: w_{1}\right]\right)=\left(\left[\xi z_{0}: z_{1}\right],\left[\xi w_{0}: w_{1}\right]\right)$, where $\xi$ is some nontrivial third root of unity. Note that this choice of coordinates does not effect the chosen shape of $\sigma$.

The branch curve $B$ of the covering $\pi: X \rightarrow Y$ is given by a $G$-(semi)-invariant polynomial $f$ of bidegree $(4,4)$. Since $\lambda$ is contained in the commutator of $G$, the polynomial $f$ is invariant with respect to the induced action of $\lambda$. In particular, it is a linear combination of $\lambda$-invariant monomials of bidegree $(4,4)$. These are

$$
z_{0}^{4} w_{0}^{2} w_{1}^{2}, z_{0}^{3} z_{1} w_{0}^{3} w_{1}, z_{0}^{3} z_{1} w_{1}^{4}, z_{0}^{2} z_{1}^{2} w_{0}^{4}, z_{0}^{2} z_{1}^{2} w_{0} w_{1}^{3}, z_{0} z_{1}^{3} w_{0}^{2} w_{1}^{2}, z_{1}^{4} w_{0}^{3} w_{1}, z_{1}^{4} w_{1}^{4}
$$

It follows that three of the four $\lambda$-fixed points on $Y$, namely ([1:0], $[1: 0]),([0: 1][1: 0])$, and ([1:0], $[0: 1]$ ) lie on $B$ and the action of $\lambda$ on the double covering $X$ has at most five fixed points. However by Remark 2.1, a symplectic action of $C_{3}$ on the K3-surface $X$ has precisely six fixed points. This yields a contradiction and we conclude $Y \not \mathbb{P}_{1} \times \mathbb{P}_{1}$.

As we have obtained contradictions for all possible choices of Del Pezzo surfaces $Y$ the non-existence claimed in Proposition 3.22 follows.

3.8. The group $N_{72}=C_{3}^{2} \rtimes D_{8}$. We let $X$ be a K3-surface with a symplectic action of $G=N_{72}$ centralized by an antisymplectic involution $\sigma$ with $\operatorname{Fix}_{X}(\sigma) \neq \emptyset$. Note that in this case we may not apply Theorem 2.10 and therefore begin by excluding that a $G$-minimal model of $Y=X / \sigma$ is an equivariant conic bundle. Then, after limiting the possible degrees of $G$-minimal models $Y_{\min }$, we study the possibility of rational branch curves.

Lemma 3.24. Any G-minimal model of $Y$ is a Del Pezzo surface.

Proof. Assume the contrary and let $Y_{\min }$ be an equivariant conic bundle and a $G$-minimal model of $Y$. We consider the induced action of $G$ on the base $\mathbb{P}_{1}$ and denote by $I \triangleleft G$ the ineffectivity of this action. Arguing as in the proof of Lemma 2.2, we see that $I$ is trivial or isomorphic to either $C_{2}$ or $C_{2} \times C_{2}$. In all cases the quotient $G / I$ contains the subgroup $C_{3} \times C_{3}$, which has no effective action on $\mathbb{P}_{1}$.

Lemma 3.25. Let $Y_{\min }$ be a Del Pezzo surface and a G-minimal model of $Y$. Then $\operatorname{deg}\left(Y_{\min }\right) \leq 4$.

Proof. We exclude all $G$-minimal Del Pezzo surfaces of degree at least five:

A Del Pezzo surface of degree five has automorphism group $S_{5}$ (cf. Theorem 10.2.2 in [Dol09]). Since $N_{72} \nless S_{5}$, it follows that $\operatorname{deg}\left(Y_{\min }\right) \neq 5$

The automorphism group of a Del Pezzo surface of degree six is $\left(\mathbb{C}^{*}\right)^{2} \rtimes\left(S_{3} \times C_{2}\right)$ (cf. Theorem 10.2.1 in [Dol09]). Assume that $N_{72}=C_{3}^{2} \rtimes D_{8}$ is contained in this group and consider the intersection $A=$ $N_{72} \cap\left(\mathbb{C}^{*}\right)^{2}$. The quotient of $N_{72}$ by $A$ is a subgroup of $S_{3} \times C_{2}$ and may not contain a copy of $C_{3}^{2}$. Therefore, the order of $A$ is at least six and $A$ contains a copy of $C_{3}$. If $|A|=6$, then $A=C_{6}=C_{3} \times C_{2}$ and $C_{2}$ is central in $N_{72}$. Using explicitly the group structure of $N_{72}$ one finds however that there is no copy of $C_{2}$ in $N_{72}$ centralizing $C_{3} \times C_{3}$. If $|A|>6$, then the centralizer of $C_{3}$ in $D_{8}$ has order greater than 2 . This is contrary to the fact that for every choice of $C_{3}$ inside $C_{3} \times C_{3}$ the centralizer inside $D_{8}$ is isomorphic to $C_{2}$. It follows that $\operatorname{deg}\left(Y_{\min }\right) \neq 6$. 
If $G$ acts on $\mathbb{P}_{1} \times \mathbb{P}_{1}$, then the canonical projections are equivariant with respect to a subgroup $H$ of index two in $G$. We consider one of these projections. The action of $H$ induces an effective action of $H / I$ on the base $\mathbb{P}_{1}$, where the group $I$ is either trivial or isomorphic to $C_{2}$ or $C_{2} \times C_{2}$. In all case we find an effective action of $C_{3}^{2}$ on the base, a contradiction.

It remains to exclude $\mathbb{P}_{2}$. If $N_{72}$ acts on $\mathbb{P}_{2}$ we consider its embedding into $\mathrm{PSL}_{3}(\mathbb{C})$, in particular the realization of the subgroup $C_{3}^{2}=\langle a\rangle \times\langle b\rangle$ and its lifting to $\mathrm{SL}_{3}(\mathbb{C})$. One uses explicit realizations of the generators $a$ and $b$ in appropriately chosen coordinates and checks that the action of $D_{8}$ on $C_{3}^{2}$ cannot be realized in $\mathrm{PSL}_{3}(\mathbb{C})$. The calculation is omitted here and the reader is referred to Section 4.8 in [Fra08] for details. It follows that there is no action of $N_{72}$ on $\mathbb{P}_{2}$.

Lemma 3.26. There are no rational curves in $\operatorname{Fix}_{X}(\sigma)$.

Proof. Let $n$ denote the total number of rational curves in $\operatorname{Fix}_{X}(\sigma)$ and recall $n \leq 10$. If $n \neq 0$, let $C$ be a rational curve in the image of $\operatorname{Fix}_{X}(\sigma)$ in $Y$ and let $H=\operatorname{Stab}_{G}(C)$ be its stabilizer. Its order is at least eight and its action on $C$ is effective. First note that $G$ does not contain $S_{4}=O_{24}$ as a subgroup. If this were the case, consider the intersection $S_{4} \cap C_{3}^{2}$ and the quotient $S_{4} \rightarrow S_{4} /\left(S_{4} \cap C_{3}^{2}\right)<D_{8}$. Since the only nontrivial normal subgroups of $S_{4}$ are $A_{4}$ and $C_{2} \times C_{2}$, this leads to a contradiction. Consequently, the order of $H$ is at most twelve. In particular, $n \geq 6$. We will obtain a contradiction by showing $n \leq 4$.

Since $|H| \geq 8$ and $C_{8} \nless G$, the group $H$ is not cyclic and any $H$-orbit on $C$ consists of at least two points. It follows from $C^{2}=-4$ that $C$ must meet the union of Mori fibers and the union of Mori fibers meets the curve $C$ in at least two points. Recalling that each Mori fibers meets the branch locus $B$ in at most two points we see that at least $n$ Mori fibers meeting $B$ are required. However, no configuration of $n$ Mori fibers is sufficient to transform the curve $C$ into a curve on a Del Pezzo surface and further Mori fibers are required. By invariance, the total number $m$ of Mori fibers must be at least $2 n$. Combining the Euler-characteristic formula (5) with our observation $\operatorname{deg}\left(Y_{\min }\right) \leq 4$, i.e., $e\left(Y_{\min }\right) \geq 8$ we see that $n \leq 4$.

Proposition 3.27. Let $X$ be a K3-surface with a symplectic action of $G=N_{72}$ centralized by an antisymplectic involution $\sigma$ with $\operatorname{Fix}_{X}(\sigma) \neq \emptyset$. Then the quotient surface $Y=X / \sigma$ is $G$-minimal and isomorphic to the Fermat cubic $\left\{x_{1}^{3}+x_{2}^{3}+x_{3}^{3}+x_{4}^{3}=0\right\} \subset \mathbb{P}_{3}$. Up to equivalence, there is a unique action of $G$ on $Y$ and the branch locus of $X \rightarrow Y$ is given by $\left\{x_{1} x_{2}+x_{3} x_{4}=0\right\}$. In particular, $X$ is equivariantly isomorphic to Mukai's $N_{72}$-example.

Proof. Using the Euler-characteristic formula (5) together with the two previous lemmata, we find that the total number $m$ of Mori fibers is bounded by four. Since the maximal order of a stabilizer group of a Mori fiber is twelve (cf. proof of Theorem 2.10) we conclude $m=0$, i.e., $Y$ must be $G$-minimal.

In order to conclude that $Y$ is the Fermat cubic we consult Dolgachev's lists of automorphisms groups of Del Pezzo surfaces of degree less than or equal to four ([Dol09] Section 10.2.2; Tables 10.3; 10,4; and 10.5): It follows immediately from the order of $G$ that $Y$ is not of degree two or four. If $G$ were a subgroup of an automorphism group of a Del Pezzo surface of degree one, it would contain a central copy of $C_{3}$, which is not the case. After excluding the cases $\operatorname{deg}(Y) \in\{1,2,4\}$ it remains to consider the case of a cubic hypersurface $Y$ in $\mathbb{P}_{3}$. The action of $G$ on $Y$ is induced by a four-dimensional (projective) representation of $G$ and the branch curve $C \subset Y$ is the intersection of $Y$ with an invariant quadric (compare proof of Proposition 3.14). It follows from the representation and invariant theory of the group $N_{72}$ that there is a unique action of $N_{72}$ on $\mathbb{P}_{3}$, a unique invariant cubic hypersurface, namely the Fermat cubic, and a unique invariant quadric hypersurface $\left\{x_{1} x_{2}+x_{3} x_{4}=0\right\}$. The necessary computations are carried out in Appendix A.1 in [Fra08].

Mukai's $N_{72}$-example is defined by $\left\{x_{1}^{3}+x_{2}^{3}+x_{3}^{3}+x_{4}^{3}=x_{1} x_{2}+x_{3} x_{4}+x_{5}^{2}=0\right\} \subset \mathbb{P}_{4}$. An anti-symplectic involution centralizing the action of $N_{72}$ is given by the map $x_{5} \mapsto-x_{5}$. The quotient of Mukai's example by this involution is the Fermat cubic and the fixed point set of the involution is given by $\left\{x_{1} x_{2}+x_{3} x_{4}=0\right\}$. 
3.9. The group $M_{9}=C_{3}^{2} \rtimes Q_{8}$. Let $G=M_{9}$ and let $X$ be a K3-surface with a symplectic $G$-action centralized by the antisymplectic involution $\sigma$ such that $\operatorname{Fix}_{X}(\sigma) \neq \emptyset$. We proceed in analogy to the case $G=N_{72}$ above. Arguing precisely as in the proof of Lemma 3.24 one shows that any $G$-minimal model of $Y$ is a Del Pezzo surface. As a next step we exclude rational branch curves.

Lemma 3.28. There are no rational curves in $\operatorname{Fix}_{X}(\sigma)$.

Proof. Let $n$ be the total number of rational curves in $\operatorname{Fix}_{X}(\sigma)$. Assume $n \neq 0$, let $C$ be a rational curve in the image of $\operatorname{Fix}_{X}(\sigma)$ in $Y$ and let $H<G$ be its stabilizer. The action of $H$ on $C$ is effective. We go through the list of finite groups with an effective action on a rational curve. Since $M_{9}$ is a group of symplectic transformations on a K3-surface, its element have order at most eight. Clearly, $A_{5}, D_{10}, D_{14}, D_{16} \nless M_{9}$. If $S_{4}<M_{9}=C_{3}^{2} \rtimes Q_{8}$, then $S_{4} \cap C_{3}^{2}$ is a normal subgroup of $S_{4}$ and it is therefore trivial. Now $S_{4}=$ $S_{4} /\left(S_{4} \cap C_{3}^{2}\right)<M_{9} / C_{3}^{2}=Q_{8}$ yields a contradiction. The same argument can be carried out for $A_{4}, D_{8}$ and $C_{8}$. If $D_{12}<M_{9}=C_{3}^{2} \rtimes Q_{8}$, then either $D_{12} \cap C_{3}^{2}=C_{3}$ and $C_{2} \times C_{2}=D_{12} / C_{3}<M_{9} / C_{3}^{2}=Q_{8}$ or $D_{12} \cap C_{3}^{2}=\{\mathrm{id}\}$ and $D_{12}<Q_{8}$, both are impossible. It follows that the subgroups of $M_{9}$ admitting an effective action on a rational curve have index greater than or equal to twelve. Therefore $n \geq 12$, contrary to inequality (3) stating $n \leq 10$.

Proposition 3.29. The quotient surface $Y$ is $G$-minimal and isomorphic to $\mathbb{P}_{2}$. Up to equivalence, there is a unique action of $G$ on $Y$ and the branch locus of $X \rightarrow Y$ is given by $\left\{x_{1}^{6}+x_{2}^{6}+x_{3}^{6}-10\left(x_{1}^{3} x_{2}^{3}+x_{2}^{3} x_{3}^{3}+x_{3}^{3} x_{1}^{3}\right)=0\right\}$. In particular, $X$ is equivariantly isomorphic to Mukai's $M_{9}$-example.

Proof. We first check that $Y$ is $G$-minimal. Again, we proceed as in the proof of Theorem 2.10 and Lemma 3.28 above to see a stabilizer group of a Mori fiber has order at most six. If $Y$ is not $G$-minimal, this implies that the total number of Mori fibers is $\geq 12$, contradicting $m \leq 9$. Note that $X \rightarrow Y$ is not branched along one or two elliptic curves as this would imply $e(Y)=12$ and contradict the fact that $Y$ is a Del Pezzo surface.

Let $B$ be the branch curve of $X \rightarrow Y$ and consider the quotient $Q$ of $B$ by the normal subgroup $N=C_{3}^{2}$ in $G$. On $Q$ there is an action of $Q_{8}$ implying that $Q$ is not rational or elliptic. It follows that the genus of $Q$ is at least two and the genus of $B$ is at least ten. Adjunction on the Del Pezzo surface $Y$ now implies $g=10$ and $Y \cong \mathbb{P}_{2}$.

It follows from direct computation involving the generators of $M_{9}$ (cf. Appendix A.2 in [Fra08]) that, up to natural equivalence, there is a unique action of $M_{9}$ on the projective plane. We may therefore consider the explicit action of $M_{9}$ on $\mathbb{P}_{2}$ specified by Mukai (cf. [Muk88]). Studying the induced action of $M_{9}$ on the space of sextic curves one finds three $M_{9}$-invariant sextic curves, namely $\left\{x_{1}^{6}+x_{2}^{6}+x_{3}^{6}-10\left(x_{1}^{3} x_{2}^{3}+\right.\right.$ $\left.\left.x_{2}^{3} x_{3}^{3}+x_{3}^{3} x_{1}^{3}\right)=0\right\}$, which is the example presented by Mukai, and additionally the two curves defined by $f_{a}=x_{1}^{6}+x_{2}^{6}+x_{3}^{6}+(18-3 a)\left(x_{1}^{2} x_{2}^{2} x_{3}^{2}\right)+2\left(x_{1}^{3} x_{2}^{3}+x_{1}^{3} x_{3}^{3}+x_{2}^{3} x_{3}^{3}\right)+a\left(x_{1}^{4} x_{2} x_{3}+x_{1} x_{2}^{4} x_{3}+x_{1} x_{2} x_{3}^{4}\right)$, where $a$ is a solution of the quadratic equation $a^{2}-6 a+36$. Depending on the choice of $a$, the polynomial $f_{a}$ is either invariant or semi-invariant with respect to the action of $M_{9}$.

We need to prove that $X$ is not the double cover of $\mathbb{P}_{2}$ branched along $\left\{f_{a}=0\right\}$. If this was the case consider the fixed point $p=[0: 1:-1]$ of the automorphism $I \in Q_{8}<M_{9}<\mathrm{PSL}_{3}(\mathbb{C})$ given by

$$
\tilde{I}=\frac{1}{\xi-\xi^{2}}\left(\begin{array}{ccc}
1 & 1 & 1 \\
1 & \xi & \xi^{2} \\
1 & \xi^{2} & \xi
\end{array}\right) \in \mathrm{SL}_{3}(\mathbb{C})
$$

for some third root of unity $\xi$ and note that $f_{a}(p)=0$. In particular, the fiber $\pi^{-1}(p)$ consists of one point $x \in X$. We linearize the $\langle I\rangle \times\langle\sigma\rangle$-action at $x$. In suitably chosen coordinates the action of the symplectic automorphism $I$ of order four is of the form $(z, w) \mapsto(i z,-i w)$. Since the action of $\sigma$ commutes with $I$, the $\sigma$-quotient of $X$ is locally given by $(z, w) \mapsto\left(z^{2}, w\right)$ or $(z, w) \mapsto\left(z, w^{2}\right)$. It follows that the action of $I$ on $Y$ is locally given by either $(x, y) \mapsto(-x,-i y)$ or $(x, y) \mapsto(i x,-y)$. In particular, the local linearization 
of $I$ at $p$ has determinant $\neq 1$. By a direct computation using the explicit form $\tilde{I} \in \mathrm{SL}_{3}(\mathbb{C})$ of the generator $I$, in particular the facts that $\operatorname{det}(\tilde{I})=1$ and $\tilde{I} v=v$ for $[v]=p$, we obtain a contradiction. This completes the proof of the proposition.

Remark 3.30. In the proof of the proposition above we have observed that an element of $\mathrm{SL}_{3}(\mathbb{C})$ does not necessarily lift to a symplectic transformation on the double cover of $\mathbb{P}_{2}$ branched along a sextic given by an invariant polynomial. Mukai's $M_{9}$-example $X$ is the double cover of $\mathbb{P}_{2}$ branched along the sextic curve $\left\{x_{1}^{6}+x_{2}^{6}+x_{3}^{6}-10\left(x_{1}^{3} x_{2}^{3}+x_{2}^{3} x_{3}^{3}+x_{3}^{3} x_{1}^{3}\right)=0\right\}$ and for this particular example, the action of $M_{9}$ does lift to a group of symplectic transformation as stated by Mukai.

To see this consider the set $\{a, b, I, J\}$ of generators of $M_{9}=C_{3}^{2} \rtimes Q_{8}=(\langle a\rangle \times\langle b\rangle) \rtimes Q_{8}$. Since $a$ and $b$ are commutators in $M_{9}$, they can be lifted to symplectic transformation $\bar{a}, \bar{b}$ on $X$. For $I, J$ consider the linearization at the fixed point $[0: 1:-1] \in \mathbb{P}_{2}$ and, using the explicit realization of the group, one checks that it has determinant one. Since $[0: 1:-1]$ is not contained in the branch set of the covering, its preimage in $X$ consists of two points $p_{1}, p_{2}$. We can lift $I(J$, respectively) to a transformation of $X$ fixing both $p_{1}, p_{2}$ and a neighbourhood of $p_{1}$ is $I$-equivariantly isomorphic to a neighbourhood of $[0: 1:-1] \in \mathbb{P}_{2}$. In particular, the action of the lifted element $\bar{I}(\bar{J}$, respectively) is symplectic. On $X$ there is the action of a degree two central extension $E$ of $M_{9}$. The elements $\bar{a}, \bar{b}, \bar{I}, \bar{J}$ generate a subgroup $\tilde{M}_{9}$ of $E_{\text {symp }}$ mapping onto $M_{9}$. Since $E_{\text {symp }} \neq E$, the order of $\tilde{M}_{9}$ is 72 and it follows that $\tilde{M}_{9}$ is isomorphic to $M_{9}$. In particular $E$ splits as $E_{\text {symp }} \times C_{2}$ with $E_{\text {symp }}=M_{9}$.

3.10. The group $T_{48}=Q_{8} \rtimes S_{3}$. We begin by specifying the group structure of $T_{48}$ : the element $c$ of order three in $S_{3}$ acts on $Q_{8}$ by permuting $I, J, K$ and an element $d$ of order two acts by exchanging $I$ and $J$ and mapping $K$ to $-K$. Now let $X$ be a K3-surface with an action of $T_{48} \times C_{2}$ where the action of $G=T_{48}$ is symplectic and the generator $\sigma$ of $C_{2}$ is antisymplectic and has fixed points.

Lemma 3.31. A G-minimal model $Y_{\min }$ of $Y$ is either $\mathbb{P}_{2}$, a Hirzebruch surface $\Sigma_{k}$ with $k>2$, or $e\left(Y_{\min }\right) \geq 9$.

Proof. Let us first consider the case where $Y_{\min }$ is a Del Pezzo surface.

If $Y_{\text {min }} \cong \mathbb{P}_{1} \times \mathbb{P}_{1}$, then both canonical projections are equivariant with respect to the index two subgroup $G^{\prime}=Q_{8} \rtimes C_{3}$. Since $Q_{8}$ has no effective action on $\mathbb{P}_{1}$, it follows that the subgroup $Z=\{+1,-1\}<Q_{8}$ acts trivially on the base. This holds with respect to both projections and the subgroup $Z$ acts trivially on $Y_{\min }$, a contradiction.

Using the group structure of $T_{48}$ one checks that the only nontrivial normal subgroup $N$ of $T_{48}$ such that $N \cap Q_{8} \neq Q_{8}$ is the center $Z=\{+1,-1\}$ of $T_{48}$. It follows that $T_{48}$ is neither a subgroup of $\left(\mathbb{C}^{*}\right)^{2} \rtimes\left(S_{3} \times C_{2}\right)$ nor a subgroup of any of the automorphism groups $C_{2}^{4} \rtimes \Gamma$ for $\Gamma \in\left\{C_{2}, C_{4}, S_{3}, D_{10}\right\}$ of a Del Pezzo surface of degree four. Furthermore, $T_{48} \nless S_{5}$ and consequently $\operatorname{deg}\left(Y_{\min }\right) \neq 4,5,6$.

So if $Y_{\min }$ is a Del Pezzo surface, then $Y_{\min } \cong \mathbb{P}_{2}$ or $e\left(Y_{\min }\right) \geq 9$.

Let us now turn to the case where $Y_{\min } \rightarrow \mathbb{P}_{1}$ is an equivariant conic bundle. The center $Z=\{+1,-1\}$ of $G=T_{48}$ acts trivially on the base and has two fixed points in the generic fiber. Let $\Lambda_{1}$ and $\Lambda_{2}$ denote the two curves of $Z$-fixed points in $Y_{\min }$.

We first show that $Y_{\min }$ is not a conic bundle with singular fibers. Any singular fiber $F$ is the union of two (-1)-curves $F_{1}, F_{2}$ meeting transversally in one point. We consider the action of $Z$ on this union of curves. The group $Z$ does not act trivially on either component of $F$ since linearization at a smooth point of $F$ would yield a trivial action of $Z$ on $Y_{\min }$. Consequently, it has either one or three fixed points on $F$. The first is impossible since $\Lambda_{1}$ and $\Lambda_{2}$ intersect $F$ in two points. It follows that $Z$ stabilizes each curve $F_{i}$. We linearize the action of $Z$ at the point of intersection $F_{1} \cap F_{2}$. The intersection is transversal and the action of $Z$ is by -Id on the tangent space $T_{F_{1}} \oplus T_{F_{2}}$ contradicting the fact the $Z$ acts trivially on the base. Thus $Y_{\min }$ is not a conic bundle with singular fibers. 
It remains to consider the case where $p: Y_{\min }=\Sigma_{k} \rightarrow \mathbb{P}_{1}$ is a Hirzebruch surface. The curves $\Lambda_{1}$ and $\Lambda_{2}$ are disjoint sections of $p$. This is only possible if $\Lambda_{1}^{2}=-\Lambda_{2}^{2}=k$. In particular, the action of $T_{48}$ on $\Sigma_{k}$ stabilizes each curve $\Lambda_{i}$. Removing the exceptional section $\Lambda_{2}=E_{\infty}$ from $\Sigma_{k}$, we obtain $H^{k}$, the $k^{\text {th }}$ power of the hyperplane bundle of $\mathbb{P}_{1}$. We chose the section $\Lambda_{2}$ to be the zero section and conclude that the action of $T_{48}$ on $H^{k}$ is by bundle automorphisms. If $k=2$, then $H^{k}$ is the anticanonical line bundle of $\mathbb{P}_{1}$ and the effective action of $S_{4}$ on the base induces an action of $S_{4}$ on $H^{2}$ by bundle automorphisms. It follows that $T_{48}$ splits as $S_{4} \times C_{2}$, a contradiction. Thus, if $Y_{\min }$ is a Hirzebruch surface $\Sigma_{k}$, then $k \neq 2$.

Lemma 3.32. There are no rational curves in $\operatorname{Fix}_{X}(\sigma)$.

Proof. We let $n$ denote the total number of rational curves in $\operatorname{Fix}_{X}(\sigma)$ and assume $n>0$. Recall $n \leq 10$, let $C$ be a rational curve in $B=\pi\left(\operatorname{Fix}_{X}(\sigma)\right) \subset Y$ and let $H=\operatorname{Stab}_{G}(C)<G$ be its stabilizer group. The action of $H$ on $C$ is effective, the index of $H$ in $G$ is at most 8. Using the quotient homomorphism $T_{48} \rightarrow T_{48} / Q_{8}=S_{3}$ one checks that $T_{48}$ does not contain $O_{24}=S_{4}$ or $T_{12}=A_{4}$ as a subgroup. It follows that $H$ is a cyclic or a dihedral group.

If $H \in\left\{C_{6}, C_{8}, D_{8}\right\}$, then $H$ and all conjugates of $H$ in $G$ contain the center $Z=\{+1,-1\}$ of $G$. It follows that $Z$ has two fixed point on each curve $g C$ for $g \in G$. Since there are six (or eight) distinct curves $g C$ in $Y$, it follows that $Z$ has at least 12 fixed points in $Y$ and in $X$. This contradicts to assumption that $Z<G$ acts symplectically on $X$ and therefore has eight fixed points in the K3-surface $X$.

It remains to study the cases $H=D_{12}$, i.e., $n=4$, and $H=D_{6}$, i.e., $n=8$.

We note that a Hirzebruch surface has precisely one curve with negative self-intersection and only fibers have self-intersection zero. A Del Pezzo surface does not contains curves of self-intersection less than -1 . The rational branch curves must therefore meet the union of Mori fibers in $Y$.

The total number of Mori fibers is bounded by $n+9$. We study the possible stabilizer subgroups $\operatorname{Stab}_{G}(E)<$ $G$ of Mori fibers. A Mori fiber $E$ with self-intersection (-1) meets the branch locus $B$ in one or two points and its stabilizer is either cyclic or dihedral. If $\operatorname{Stab}_{G}(E) \in\left\{C_{4}, D_{8}\right\}$, then the points of intersection of $E$ and $B$ are fixed points of the center $Z$ of $G$ and we find too many $Z$-fixed points on $X$.

Assume $n=4$ and let $R_{1}, \ldots R_{4}$ be the rational curves in $B$. We denote by $\tilde{R}_{i}$ their images in $Y_{\min }$. The total number $m$ of Mori fibers is bounded by 12. We go through the list of possible configurations:

- If $m=4$, there is no invariant configuration of Mori fibers such that the contraction maps the four rational branch curves to an admissible configuration on the Hirzebruch or Del Pezzo surface $Y_{\min }$.

- If $m=6$, then $\operatorname{Stab}_{G}(E)=C_{8}$ and the points of intersection of $E$ and $B$ are $Z$-fixed. Since $Z$ has at most eight fixed points on $B$, it follows that each curve $E$ meets $B$ only once. The images $\tilde{R}_{i}$ of the $R_{i}$ contradict our observations about curves in Del Pezzo and Hirzebruch surfaces.

- If $m=8$ and all Mori fibers have self-intersection -1 , then each Mori fiber meets $\bigcup R_{i}$ in a $Z$-fixed point. Since there at at most eight such points, it follows that each Mori fibers meets $\bigcup R_{i}$ only once and their contractions does not transform the curves $R_{i}$ sufficiently.

- If $m=8$ and only four Mori fibers have self-intersection -1 , we consider the four Mori fibers of the second reduction step. Each of these meets a Mori fiber $E$ of the first step in precisely one point. By invariance, this would have to be a fixed points of the stabilizer $\operatorname{Stab}_{G}(E)=D_{12}$, a contradiction.

- If $m=12$, then either $e\left(Y_{\min }\right)=3$ and there exist a branch curve $B_{g=2}$ of genus two or $e\left(Y_{\min }\right)=4$ and $B=\bigcup R_{i}$. In the first case, $Y_{\min } \cong \mathbb{P}_{2}$ and twelve Mori fibers are not sufficient to transform $B=B_{g=2} \cup \bigcup R_{i}$ into an admissible configuration of curves in the projective plane. So $Y_{\min }=\Sigma_{k}$ for $k>2$. Recall that $Z$ has two fixed points in each fiber of $p: \Sigma_{k} \rightarrow \mathbb{P}_{1}$, i.e., the $Z$-action on $\Sigma_{k}$ has two disjoint curves of fixed points. As was remarked above, these curves are the exceptional section $E_{\infty}$ of self-intersection $-k$ and a section $E_{0} \sim E_{\infty}+k F$ of self-intersection $k$. Here $F$ denotes a fiber of $p: \Sigma_{k} \rightarrow \mathbb{P}_{1}$. There is no automorphisms of $\Sigma_{k}$ mapping $E_{\infty}$ to $E_{0}$. Each rational branch 
curve $\tilde{R}_{i}$ has two $Z$-fixed points. These are exchanged by an element of $\operatorname{Stab}_{G}\left(R_{i}\right)$ and therefore both lie on either $E_{\infty}$ or $E_{0}$, i.e., $\tilde{R}_{i}$ cannot have nontrivial intersection with both $E_{0}$ and $E_{\infty}$. By invariance all curves $\tilde{R}_{i}$ either meet $E_{0}$ or $E_{\infty}$ and not both. Using the fact that $\sum \tilde{R}_{i}$ is linearly equivalent to $-2 K_{\Sigma_{k}} \sim 4 E_{\infty}+(2 k+4) F$ we find that $\tilde{R}_{i} \cdot E_{\infty}=0$ and $k=2$, a contradiction to Lemma 3.31 .

We have shown that all possible configurations in the case $n \neq 4$ lead to a contradiction. We now turn to the case $n=8$ and let $R_{1}, \ldots R_{8}$ be the rational branch curves. The total number of Mori fibers is bounded by 16 . Note that by invariance, the $G$-orbit of a Mori fiber meets $\bigcup R_{i}$ in at least 16 points or not at all. In particular, Mori fibers meeting $R_{i}$ come in orbits of length $\geq 8$. As above, we go through the list of possible configurations.

- If $m=16$, then the set of all Mori fibers consists of one orbit of length 16 or of two orbits of length eight. If all 16 Mori fibers meet $B$, then each meets $B$ in one point and $R_{i}$ is mapped to a (-2)-curve in $Y_{\min }$. If only eight Mori fibers meet $B$, then each of the eight Mori fibers of the second reduction step meets one Mori fiber $E$ of the first reduction step in one point. This point has to be a $\operatorname{Stab}_{G}(E)$-fixed point. The $\operatorname{Stab}_{G}(E)$-fixed points on $E$ must however coincide with the points $E \cap B$, a contradiction.

- If $m=12$, then the set of all Mori fibers consists of a single $G$-orbit and each curve $R_{i}$ meets three distinct Mori fibers. Their contraction transforms $R_{i}$ into a (-1)-curve on $Y_{\min }$. It follows that $Y_{\min }$ contains at least eight $(-1)$-curves and is a Del Pezzo surface of degree $\leq 5$. We have seen above that $\operatorname{deg}\left(Y_{\min }\right) \neq 4,5$ and therefore $e\left(Y_{\min }\right) \geq 9$. With $m=12$ and $n=8$, this contradicts the Euler characteristic formula $24=2 e\left(Y_{\min }\right)+2 m-2 n+(2 g-2)$.

- If $m=8$ there is no invariant configuration of Mori fibers such that the contraction maps the eight rational branch curves to an admissible configuration on the Hirzebruch or Del Pezzo surface $Y_{\min }$.

This completes the proof of the lemma.

Since there is an effective action of $T_{48}$ on $\operatorname{Fix}_{X}(\sigma)$, it is neither an elliptic curve nor the union of two elliptic curves. It follows that $X \rightarrow Y$ is branched along a single $T_{48}$-invariant curve $B$ with $g(B) \geq 2$.

Lemma 3.33. The genus of $B$ is neither three nor four.

Proof. We consider the quotient $Q=B / Z$ of the curve $B$ by the center $Z$ of $G$ and apply the Euler characteristic formula, $e(B)=2 e(Q)-\left|F_{x_{B}}(Z)\right|$. On $Q$ there is an effective action of the group $G / Z=\left(C_{2} \times C_{2}\right) \rtimes C_{3}=$ $S_{4}$. Using Remark 3.19 we see that $e(Q) \in\{2,-4,-6,-8 \ldots\}$.

If $g(B)=3$, then $e(B)=-4$ and the only possibility is $Q \cong \mathbb{P}_{1}$ and $\left|F_{B}(Z)\right|=8$. In particular, all $Z$-fixed points on $X$ are contained in the curve $R=\pi^{-1}(B)$. Let $A<G$ be the group generated by $I \in Q_{8}=$ $\{ \pm 1, \pm I, \pm J, \pm K\}$. The four fixed points of $A$ in $X$ are contained in $\operatorname{Fix}_{X}(Z)=\operatorname{Fix}_{B}(Z)$ and the quotient group $A / Z \cong C_{2}$ has four fixed points in $Q$. This is a contradiction.

If $g(B)=4$, then $e(B)=-6$ and the only possibility is $Q \cong \mathbb{P}_{1}$ and $\left|\operatorname{Fix}_{B}(Z)\right|=10$. This contradicts the fact that $Z$ has at most eight fixed points in $B$ since it has precisely eight fixed points in $X$.

In Lemma 3.31 we have reduced the classification to the cases $e\left(Y_{\min }\right) \in\{3,4,9,10,11\}$. In the following, we will exclude the cases $e\left(Y_{\min }\right) \in\{4,9,10\}$ and describe the remaining cases more precisely. Recall that the maximal possible stabilizer subgroup of a Mori fiber is $D_{12}$, in particular, $m=0$ or $m \geq 4$.

Lemma 3.34. If $e\left(Y_{\min }\right)=3$, then $Y_{\min }=Y=\mathbb{P}_{2}$ and $X \rightarrow Y$ is branched along the curve $\left\{x_{1} x_{2}\left(x_{1}^{4}-x_{2}^{4}\right)+\right.$ $\left.x_{3}^{6}=0\right\}$. In particular, $Y$ is equivariantly isomorphic to Mukai's $T_{48}$-example. 
Proof. Let $M: Y \rightarrow \mathbb{P}_{2}$ denote a Mori reduction of $Y$ and let $B \subset Y$ be the branch curve of the covering $X \rightarrow Y$. If $Y=Y_{\min }$, then $B=M(B)$ is a smooth sextic curve. If $Y \neq Y_{\min }$, then the Euler characteristic formula with $m \in\{4,6,8\}$ shows that $g(B) \in\{2,4,6\}$. The case $m=6, g(B)=4$ has been excluded by the previous lemma.

If $m=4$, then the stabilizer group of each Mori fiber is $D_{12}$ and each Mori fiber meets $B$ in two points. The image $M(B)$ of $B$ in $Y_{\min }$ is an irreducible singular sextic.

If $m=8$, then $g(B)=2$ and $B^{2}=4$. Since the self-intersection number $M(B)^{2}$ must be a square, one checks that all possible invariant configurations of Mori fibers yield $M(B)^{2}=36$ and involve Mori fibers meeting $B$ in two points. In particular, also in this case $M(B)$ must be a singular sextic.

By explicit computation using the group structure of $T_{48}$ one can determine the unique action of $T_{48}$ on $\mathbb{P}_{2}$. This is carried out in detail in Section 4.10 in [Fra08]. Using the explicit form of the $T_{48}$-action and the fact that the commutator subgroup of $T_{48}$ is $Q_{8} \rtimes C_{3}$ one then checks that any invariant curve of degree six is of the form

$$
C_{\lambda}=\left\{x_{1} x_{2}\left(x_{1}^{4}-x_{2}^{4}\right)+\lambda x_{3}^{6}=0\right\}
$$

for some $\lambda \in \mathbb{C}^{*}$. In order to avoid this calculation, one can also argue that the polynomial $x_{1} x_{2}\left(x_{1}^{4}-x_{2}^{4}\right)$ is the lowest order invariant of the octahedral group $S_{4} \cong T_{48} / Z$. The curve $C_{\lambda}$ is smooth and it follows that $Y=Y_{\min }$. We may adjust the coordinates equivariantly such that $\lambda=1$ and find that our surface $X$ is precisely Mukai's $T_{48}$-example.

Remark 3.35. As stated by Mukai, the action of $T_{48}$ on $\mathbb{P}_{2}$ does indeed lift to a symplectic action of $T_{48}$ on the double cover of $\mathbb{P}_{2}$ branched along the invariant curve $\left\{x_{1} x_{2}\left(x_{1}^{4}-x_{2}^{4}\right)+x_{3}^{6}=0\right\}$. The elements of the commutator subgroup can be lifted to symplectic transformation on the double cover $X$. The remaining generator $d$ is an involution fixing the point $[0: 0: 1]$. Any involution $\tau$ with a fixed point $p$ outside the branch locus can be lifted to a symplectic involution on the double cover $X$ as follows: The linearized action of $\tau$ at $p$ has determinant \pm 1 . We consider the lifting $\tilde{\tau}$ of $\tau$ fixing both points in the preimage of $p$. Its linearization coincides with the linearization on the base and therefore also has determinant \pm 1 . In particular, $\tilde{\tau}$ is an involution. It follows that either $\tilde{\tau}$ or the second choice of a lifting $\sigma \tilde{\tau}$ acts symplectically on $X$. The group generated by all lifted automorphisms is either isomorphic to $T_{48}$ or to its degree two central extension $E$ acting on the double cover. Since $E_{\text {symp }} \neq E$ the later is impossible it follows that $E$ splits as $E_{\text {symp }} \times C_{2}$ with $E_{\text {symp }}=T_{48}$.

Finally, we return to the remaining possibilities $e\left(Y_{\min }\right) \in\{4,9,10,11\}$.

Lemma 3.36. $e\left(Y_{\min }\right) \notin\{4,9,10\}$.

Proof. Recalling that the genus of the branch curve $B$ is neither three nor four and that $m$ is either zero or $\geq 4$, we may exclude $e\left(Y_{\min }\right)=9,10$ using the Euler characteristic formula $12=e\left(Y_{\min }\right)+m+g-1$. It remains to consider the case $Y_{\min }=\Sigma_{k}$ with $k>2$ and we claim that this is impossible.

Let $M: Y \rightarrow Y_{\min }=\Sigma_{k}$ denote a (possibly trivial) Mori reduction of $Y$. The image $M(B)$ of $B$ in $\Sigma_{k}$ is linearly equivalent to $-2 K_{\Sigma_{k}}$. Now $M(B) \cdot E_{\infty}=2(2-k)<0$ and it follows that $M(B)$ contains the rational curve $E_{\infty}$. This is a contradiction since $B$ does not contain any rational curves by Lemma 3.32 .

In the last remaining case, i.e., $e\left(Y_{\min }\right)=11$, the quotient surface $Y$ is a $G$-minimal Del Pezzo surface of degree 1. Consulting [Dol09], Table 10.5, we find that $Y$ is a hypersurface in weighted projective space $\mathbb{P}(1,1,2,3)$ defined by the weighted homogeneous equation $x_{1} x_{2}\left(x_{1}^{4}-x_{2}^{4}\right)+x_{3}^{3}+x_{4}^{2}$. This follows from the invariant theory of the group $S_{4} \cong T_{48} / Z$ and fact that the anticanonical map realizes $Y$ as a double cover of a quadric cone $Q$ in $\mathbb{P}_{3}$ branched along the intersection of $Q$ with a cubic hypersurface.

The linear system of the anticanonical divisor $K_{Y}$ has precisely one base point $p$. In coordinates $\left[x_{1}: x_{2}\right.$ : $\left.x_{3}: x_{4}\right]$ this point is given as $[0: 0: 1: i]$. It is fixed by the action of $T_{48}$. The linearization of $T_{48}$ 
at $p$ is given by the unique faithful 2-dimensional represention of $T_{48}$. It follows that there is a unique action of $T_{48}$ on $Y$. The branch curve $B$ is linearly equivalent to $-2 K_{Y}$, i.e., $B=\{s=0\}$ for a section $s \in \Gamma\left(Y, \mathscr{O}\left(-2 K_{Y}\right)\right)$ which is either invariant or semi-invariant.

The adjunction formula for hypersurfaces in weighted projective space (cf. Theorem 3.3.4 in [Dol82]) yields $\left.\mathscr{O}\left(-2 K_{Y}\right)\right)=\mathscr{O}_{Y}(2)$. The four-dimensional space of sections $\Gamma\left(Y, \mathscr{O}\left(-2 K_{Y}\right)\right)$ is generated by the weighted homogeneous polynomials $x_{1}^{2}, x_{2}^{2}, x_{1} x_{2}, x_{3}$. We consider the map $Y \rightarrow \mathbb{P}\left(\Gamma\left(Y, \mathscr{O}\left(-2 K_{Y}\right)\right)^{*}\right)$ associated to $\left|-2 K_{Y}\right|$. Since this map is equivariant with respect to $\operatorname{Aut}(Y)$, the fixed point $p$ is mapped to a fixed point in $\mathbb{P}\left(\Gamma\left(Y, \mathscr{O}\left(-2 K_{Y}\right)\right)^{*}\right)$. It follows that the section corresponding to the homogeneous polynomial $x_{3}$ is invariant or semi-invariant with respect to $T_{48}$. It is the only section of $\mathscr{O}\left(-2 K_{Y}\right)$ with this property since the representation of $T_{48}$ on the span of $x_{1}^{2}, x_{2}^{2}, x_{1} x_{2}$ is irreducible. It follows that $B=\left\{x_{3}=0\right\}$.

In order to prove existence, let $b: Y \rightarrow \mathbb{P}_{2}$ be the blow down of eight disjoint (-1)-curves in $Y$. The curve $C=b(B)$ is a singular sextic. The double cover $X$ of $Y$ branched along $B$ is the minimal desingularization of the double cover $X_{\text {sing }}$ of $\mathbb{P}_{2}$ branched along $C$. In particular, one finds that $X$ is a K3-surface. It remains to check that the action of $T_{48}$ on $Y$ lifts to a group of symplectic transformation on $X$. First note that $B$ does not contain the base point $p$. For $I, J, K, c \in T_{48}$ we choose liftings $\bar{I}, \bar{J}, \bar{K}, \bar{c} \in \operatorname{Aut}(X)$ fixing both points in $\pi^{-1}(p)=\left\{p_{1}, p_{2}\right\}$. The linearization of $\bar{I}, \bar{J}, \bar{K}, \bar{c}$ at $p_{1}$ is the same as the linearization at $p$ and in particular has determinant one. By the general considerations in Remark 3.35 the involution $d$ can be lifted to a symplectic involution on $X$. The symplectic liftings of $I, J, K, c, d$ generate a subgroup $\tilde{G}$ of $\operatorname{Aut}(X)$ which is isomorphic to either $T_{48}$ or to the central degree two extension of $T_{48}$ acting on $X$. In analogy to Remarks 3.30 and 3.35 we conclude that $\tilde{G} \cong T_{48}$ and the action of $T_{48}$ on $Y$ induces a symplectic action of $T_{48}$ on the double cover $X$.

This completes the classification of K3-surfaces with $T_{48} \times C_{2}$-symmetry. We have shown:

Proposition 3.37. Let $X$ be a K3-surface with a symplectic action of the group $T_{48}$ centralized by an antisymplectic involution $\sigma$ with $\operatorname{Fix}_{X}(\sigma) \neq \emptyset$. Then $X$ is equivariantly isomorphic either to Mukai's $T_{48}$-example or to the double cover of $\left\{x_{1} x_{2}\left(x_{1}^{4}-x_{2}^{4}\right)+x_{3}^{3}+x_{4}^{2}=0\right\} \subset \mathbb{P}(1,1,2,3)$ branched along $\left\{x_{3}=0\right\}$

Remark 3.38. The automorphism group of the Del Pezzo surface $Y=\left\{x_{1} x_{2}\left(x_{1}^{4}-x_{2}^{4}\right)+x_{3}^{3}+x_{4}^{2}=0\right\} \subset$ $\mathbb{P}(1,1,2,3)$ is the trivial central extension $C_{3} \times T_{48}$. By contruction, the curve $B=\{s=0\}$ is invariant with respect to the full automorphism group. The double cover $X$ of $Y$ branched along $B$ carries the action of a finite group $\tilde{G}$ of order $2 \cdot 3 \cdot 48=288$ containing $T_{48}<\tilde{G}_{\text {symp }}$. Since $T_{48}$ is a maximal group of symplectic transformations, we find $T_{48}=\tilde{G}_{\text {symp. }}$. In analogy to the proof of Claim 2.1 in [OZ02], one can check that 288 is the maximal order of a finite group $H$ acting on a K3-surface with $T_{48}<H_{\text {symp }}$. It follows that $\tilde{G}$ is a maximal finite subgroup of $\operatorname{Aut}(X)$.

Conclusion. The classification and non-existence results for the individual groups from Table 1 obtained in the preceding subsections yield the proof of Theorem 3.1 .

\section{REFERENCES}

[BaBe00] Lionel Bayle and Arnaud Beauville, Birational involutions of $\mathbf{P}^{2}$, Asian J. Math. 4 (2000), no. 1, 11-17, Kodaira's issue.

[Bea07] Arnaud Beauville, p-elementary subgroups of the Cremona group, J. Algebra 314 (2007), no. 2, 553-564.

[BeB104] Arnaud Beauville and Jérémy Blanc, On Cremona transformations of prime order, C. R. Math. Acad. Sci. Paris 339 (2004), no. 4, 257-259.

[Bla06] Jérémy Blanc, Finite Abelian subgroups of the Cremona group of the plane, Ph.D. thesis, Université de Genève, http://arxiv.org/abs/math.AG/0610368 2006.

[Bla07] - Finite abelian subgroups of the Cremona group of the plane, C. R. Math. Acad. Sci. Paris 344 (2007), no. 1, 21-26.

[Bli17] Hans Frederik Blichfeldt, Finite collineation groups, The University of Chicago Press, Chicago, 1917.

[Cra99] Scott Crass, Solving the sextic by iteration: a study in complex geometry and dynamics, Experiment. Math. 8 (1999), no. 3, 209-240.

[dF04] Tommaso de Fernex, On planar Cremona maps of prime order, Nagoya Math. J. 174 (2004), 1-28. 
[DI06] Igor V. Dolgachev and Vasily A. Iskovskikh, Finite subgroups of the plane Cremona group, to appear in Algebra, Arithmetic, and Geometry, Volume I: In Honor of Y.I. Manin, Progress in Mathematics, http://arxiv.org/abs/math/0610595 2006.

[DI07] On elements of prime order in the plane Cremona group over a perfect field, to appear in Int. Math. Res. Not., http://arxiv.org/abs/0707.4305 2007.

[Dol82] Igor V. Dolgachev, Weighted projective varieties, Group actions and vector fields (Vancouver, B.C., 1981), Lecture Notes in Math., vol. 956, Springer, Berlin, 1982, 34-71.

[Dol09] _ Topics in classical algebraic geometry. Part I, http://www.math.1sa.umich.edu/ idolga/topics1.pdf 2009.

[FH08] Kristina Frantzen and Alan Huckleberry, K3-surfaces with special symmetry: An example of classification by Mori-reduction, Complex Geometry in Osaka, In honour of Professor Akira Fujiki on the occasion of his 60th birthday, 2008, 86-99.

[FH09] _ Finite symmetry groups in complex geometry, Journées Élie Cartan 2006, 2007 et 2008 - Nancy, Revue de l'Institute Élie Cartan 19 (2009), 73-113.

[Fra08] Kristina Frantzen, K3-surfaces with special symmetry, Ph.D. thesis, Ruhr-Universität Bochum, http://arxiv.org/abs/0902.3761.2008.

[Isk80] Vasily A. Iskovskikh, Minimal models of rational surfaces over arbitrary fields, Math. USSR-Izv. 14 (1980), no. 1, 17-39.

[KM98] János Kollár and Shigefumi Mori, Birational geometry of algebraic varieties, Cambridge Tracts in Mathematics, vol. 134, Cambridge University Press, Cambridge, 1998.

[Kon98] Shigeyuki Kondō, Niemeier lattices, Mathieu groups, and finite groups of symplectic automorphisms of K3 surfaces, Duke Math. J. 92 (1998), no. 3, 593-603, With an appendix by Shigeru Mukai.

[KOZ05] JongHae Keum, Keiji Oguiso, and De-Qi Zhang, The alternating group of degree 6 in the geometry of the Leech lattice and K3 surfaces, Proc. London Math. Soc. (3) 90 (2005), no. 2, 371-394.

[KOZ07] _ Extensions of the alternating group of degree 6 in the geometry of K3 surfaces, European J. Combin. 28 (2007), no. 2, 549-558.

[Man67] Yuri I. Manin, Rational surfaces over perfect fields. II, Math. USSR-Sb. 1 (1967), no. 2, 141-168.

[MBD16] George Abram Miller, Hans Frederik Blichfeldt, and Leonard Eugene Dickson, Theory and applications of finite groups, Dover, New York, 1916.

[Mor82] Shigefumi Mori, Threefolds whose canonical bundles are not numerically effective, Ann. of Math. (2) 116 (1982), no. 1, 133-176.

[Muk88] Shigeru Mukai, Finite groups of automorphisms of K3 surfaces and the Mathieu group, Invent. Math. 94 (1988), no. 1, 183-221.

[Nik76] Viacheslav V. Nikulin, Kummer surfaces, Math. USSR. Izv. 9 (1976), no. 2, 261-275.

[Nik80] _ Finite automorphism groups of Kähler K3 surfaces, Trans. Moscow Math. Soc 38 (1980), no. 2, 71-135.

[Nik83] - On factor groups of groups of automorphisms of hyperbolic forms with respect to subgroups generated by 2-reflections. algebrogeometric applications, J. Soviet Math. 22 (1983), 1401-1476.

[OZ02] Keiji Oguiso and De-Qi Zhang, The simple group of order 168 and K3 surfaces, Complex geometry (Göttingen, 2000), Springer, Berlin, 2002, 165-184.

[YY93] Stephen S.-T. Yau and Yung Yu, Gorenstein quotient singularities in dimension three, Mem. Amer. Math. Soc. 105 (1993), no. 505.

[Zha98] De-Qi Zhang, Quotients of K3 surfaces modulo involutions, Japan. J. Math. (N.S.) 24 (1998), no. 2, 335-366.

[Zha01] _ Automorphisms of finite order on rational surfaces, J. Algebra 238 (2001), no. 2, 560-589, With an appendix by Igor V. Dolgachev.

[Zha07] _ Automorphisms of K3 surfaces, Proceedings of the International Conference on Complex Geometry and Related Fields (Providence, RI), AMS/IP Stud. Adv. Math., vol. 39, Amer. Math. Soc., 2007, 379-392. 